\title{
CALIBRATING EXTINCTION-FREE STAR FORMATION RATE DIAGNOSTICS WITH 33 GHz FREE-FREE EMISSION IN NGC 6946
}

\author{
E. J. Murphy ${ }^{1}$, J. J. Condon ${ }^{2}$, E. Schinnerer ${ }^{3}$, R. C. Kennicutt, Jr. ${ }^{4}$, D. Calzettio ${ }^{5}$, L. Armus ${ }^{1}$, G. Helou ${ }^{6}$, J. L. Turner ${ }^{7}$, \\ G. Aniano ${ }^{8}$, P. Beirão ${ }^{1}$, A. D. Bolatto ${ }^{9}$, B. R. Brandl ${ }^{10}$, K. V. Croxall ${ }^{11}$, D. A. Dale ${ }^{12}$, J. L. Donovan Meyer ${ }^{13}$, \\ B. T. Draine ${ }^{8}$, C. Engelbracht ${ }^{14}$, L. K. Hunt ${ }^{15}$, C.-N. HaO ${ }^{16}$, J. Koda ${ }^{13}$, H. Roussel ${ }^{17}$, R. Skibba ${ }^{14}$, and J.-D. T. Smith ${ }^{11}$ \\ ${ }^{1}$ Spitzer Science Center, California Institute of Technology, MC 314-6, Pasadena, CA 91125, USA; emurphy@ipac.caltech.edu \\ 2 National Radio Astronomy Observatory, Charlottesville, VA 22903, USA \\ ${ }^{3}$ Max-Planck-Institut für Astronomie, Heidelberg D-69117, Germany \\ ${ }^{4}$ Institute of Astronomy, University of Cambridge, Cambridge, CB3 OHA, UK \\ ${ }^{5}$ Department of Astronomy, University of Massachusetts, Amherst, MA 01003, USA \\ ${ }^{6}$ California Institute of Technology, MC 314-6, Pasadena, CA 91125, USA \\ ${ }^{7}$ Department of Physics and Astronomy, UCLA, Los Angeles, CA 90095, USA \\ ${ }^{8}$ Department of Astrophysical Sciences, Princeton University, Princeton, NJ 08544, USA \\ ${ }^{9}$ Department of Astronomy, University of Maryland, College Park, MD 20742, USA \\ ${ }^{10}$ Leiden Observatory, Leiden University, 2300 RA Leiden, The Netherlands \\ ${ }^{11}$ Department of Physics and Astronomy, University of Toledo, Toledo, OH 43606, USA \\ 12 Department of Physics \& Astronomy, University of Wyoming, Laramie, WY 82071, USA \\ ${ }^{13}$ Department of Physics and Astronomy, SUNY Stony Brook, Stony Brook, NY 11794-3800, USA \\ ${ }^{14}$ Steward Observatory, University of Arizona, Tucson, AZ 85721, USA \\ 15 INAF-Osservatorio Astrofisico di Arcetri, 50125 Firenze, Italy \\ 16 Tianjin Astrophysics Center, Tianjin Normal University, Tianjin 300387, China \\ ${ }^{17}$ Institut d'Astrophysique de Paris, UMR7095 CNRS Université Pierre \& Marie Curie, 75014 Paris, France \\ Received 2011 February 9; accepted 2011 May 24; published 2011 August 4
}

\begin{abstract}
Using free-free emission measured in the Ka band (26-40 GHz) for 10 star-forming regions in the nearby galaxy NGC 6946, including its starbursting nucleus, we compare a number of star formation rate (SFR) diagnostics that are typically considered to be unaffected by interstellar extinction. These diagnostics include non-thermal radio (i.e., 1.4 GHz), total infrared (IR; 8-1000 $\mu \mathrm{m}$ ), and warm dust (i.e., $24 \mu \mathrm{m}$ ) emission, along with hybrid indicators that attempt to account for obscured and unobscured emission from star-forming regions including $\mathrm{H} \alpha+24 \mu \mathrm{m}$ and $\mathrm{UV}+\mathrm{IR}$ measurements. The assumption is made that the $33 \mathrm{GHz}$ free-free emission provides the most accurate measure of the current SFR. Among the extranuclear star-forming regions, the $24 \mu \mathrm{m}, \mathrm{H} \alpha+24 \mu \mathrm{m}$, and UV + IR SFR calibrations are in good agreement with the $33 \mathrm{GHz}$ free-free SFRs. However, each of the SFR calibrations relying on some form of dust emission overestimates the nuclear SFR by a factor of $\sim 2$ relative to the $33 \mathrm{GHz}$ free-free SFR. This is more likely the result of excess dust heating through an accumulation of non-ionizing stars associated with an extended episode of star formation in the nucleus rather than increased competition for ionizing photons by dust. SFR calibrations using the non-thermal radio continuum yield values which only agree with the $33 \mathrm{GHz}$ free-free SFRs for the nucleus and underestimate the SFRs from the extranuclear star-forming regions by an average factor of $\sim 2$ and $\sim 4-5$ before and after subtracting local background emission, respectively. This result likely arises from the cosmic-ray (CR) electrons decaying within the starburst region with negligible escape, whereas the transient nature of star formation in the young extranuclear star-forming complexes allows for CR electrons to diffuse significantly further than dust-heating photons, resulting in an underestimate of the true SFR. Finally, we find that the SFRs estimated using the total $33 \mathrm{GHz}$ flux density appear to agree well with those estimated using free-free emission due to the large thermal fractions present at these frequencies even when local diffuse backgrounds are not removed. Thus, rest-frame $33 \mathrm{GHz}$ observations may act as a reliable method to measure the SFRs of galaxies at increasingly high redshift without the need of ancillary radio data to account for the non-thermal emission.
\end{abstract}

Key words: cosmic rays - galaxies: individual (NGC 6946) - H II regions - infrared: general - radio continuum: general - stars: formation

\section{INTRODUCTION}

Accurate estimates of star formation rates (SFRs) are crucial in nearly all fields of extragalactic astronomy. Besides requiring a high level of accuracy, the ease with which such estimates can be made plays a critical role in the overall utility of an SFR diagnostic given that, for studying galaxy evolution, observations need to be made over large areas of sky and be deep enough to detect galaxies at high redshifts. Presently, a wide variety of SFR diagnostics are commonly used in the literature (e.g., Kennicutt 1998), each of which has its strengths and shortcomings.
Rest-frame UV (1250-2500 ̊̊) and optical (e.g., H $\alpha$ ) observations provide a measure of the radiant energy released by newly formed massive stars. While directly related to the massive star formation process, such observations are hampered by interstellar extinction that varies enormously within and among galaxies. This is especially problematic for trying to characterize the evolution of the SFR density with redshift as the galaxies that dominate the stellar mass assembly between $1 \lesssim z \lesssim 3$ appear to be dusty starbursts with infrared (IR; 8-1000 $\mu \mathrm{m}$ ) luminosities ranging between $10^{10} L_{\odot} \lesssim L_{\mathrm{IR}} \lesssim 10^{13} L_{\odot}$ (e.g., Chary \& Elbaz 2001; Takeuchi et al. 2005; Bouwens et al. 2009; Murphy et al. 2011). In these IR-bright starbursts, more than $\gtrsim 90 \%$ of 
the UV emission can be obscured (e.g., Howell et al. 2010; Buat et al. 2010; Murphy et al. 2011).

Rest-frame IR measurements, particularly those in the farinfrared (FIR; 42.5-122.5 $\mu \mathrm{m}$ ), do not suffer significantly from extinction as they probe the re-radiated $\mathrm{UV} /$ optical emission from heated dust grains surrounding massive star-forming regions. While an extinction-free measure, the interpretation of IR emission alone is also complex. Variations in the dust composition, content, and distribution along the line of sight will affect the fraction of UV photons absorbed, while a portion of the IR emission will arise from dust heated by older stars (i.e., the cirrus component; Helou 1986; Lonsdale Persson \& Helou 1987; Hirashita et al. 2003; Bendo et al. 2010; Li et al. 2010).

Radio continuum emission is also widely used as a tracer of star formation in galaxies both at low and high redshifts as the result of the tight empirical correlation between their optically thin synchrotron emission at $1.4 \mathrm{GHz}$ and their FIR radiation (e.g., de Jong et al. 1985; Helou et al. 1985). While this relation (i.e., the FIR-radio correlation) is certainly rooted in common dependencies on massive star formation, it is unclear how presumably unrelated physical processes affecting the propagation of cosmic-ray (CR) electrons and the heating of dust grains work together to yield a nearly ubiquitous correlation over nearly five orders of magnitude in luminosity (Yun et al. 2001). Spitzer revealed local correlations in the spatial distributions of $70 \mu \mathrm{m}$ and non-thermal radio emission in the disks of galaxies that reflect an age effect indicating the complexities of using non-thermal radio continuum emission as an SFR diagnostic (Murphy et al. 2006b, 2008). Additional complications for using the non-thermal radio emission as an SFR diagnostic are also evident given that the correlation between the IR and radio emission is strikingly different for spiral arms and interarm regions (Dumas et al. 2011).

Higher-frequency radio continuum emission, which becomes dominated by thermal (free-free) radiation beyond $\sim 30 \mathrm{GHz}$ for globally integrated measurements of star-forming galaxies (e.g., Condon 1992), is largely extinction free and can be directly related to the ionizing photon rate arising from newly formed massive stars. Evidence for this has been indicated by detailed studies of star-forming regions in the Galaxy (e.g., Mezger \& Henderson 1967), nearby dwarf irregulars (e.g., Klein \& Graeve 1986), the nuclei of normal galaxies (e.g., Turner \& Ho 1983, 1994), and starbursts (e.g., Klein et al. 1988; Turner \& Ho 1985), as well as high-resolution investigations of super star clusters within nearby blue compact dwarfs (e.g., Turner et al. 1998; Kobulnicky \& Johnson 1999).

While typically considered an ideal measure for the current star formation activity in galaxies, the presence of an "anomalous" dust emission component in excess of the free-free emission between $\sim 10$ and $90 \mathrm{GHz}$, generally attributed to rapidly rotating ultrasmall grains with a non-zero electric moment (e.g., Draine \& Lazarian 1998; Planck Collaboration et al. 2011), may complicate this picture. For a single outer-disk star-forming region in NGC 6946, Murphy et al. (2010) detected excess $33 \mathrm{GHz}$ emission relative to what is expected given existing lower frequency radio data. This result is interpreted as the first likely detection of so-called anomalous dust emission outside of the Milky Way. Given that the excess was only detected for a single region, this emission component may be negligible for globally integrated measurements.

In this paper, we quantitatively compare SFR estimates using a number of "extinction-free" diagnostics for 10 star-forming regions within the nearby galaxy NGC 6946 presented in

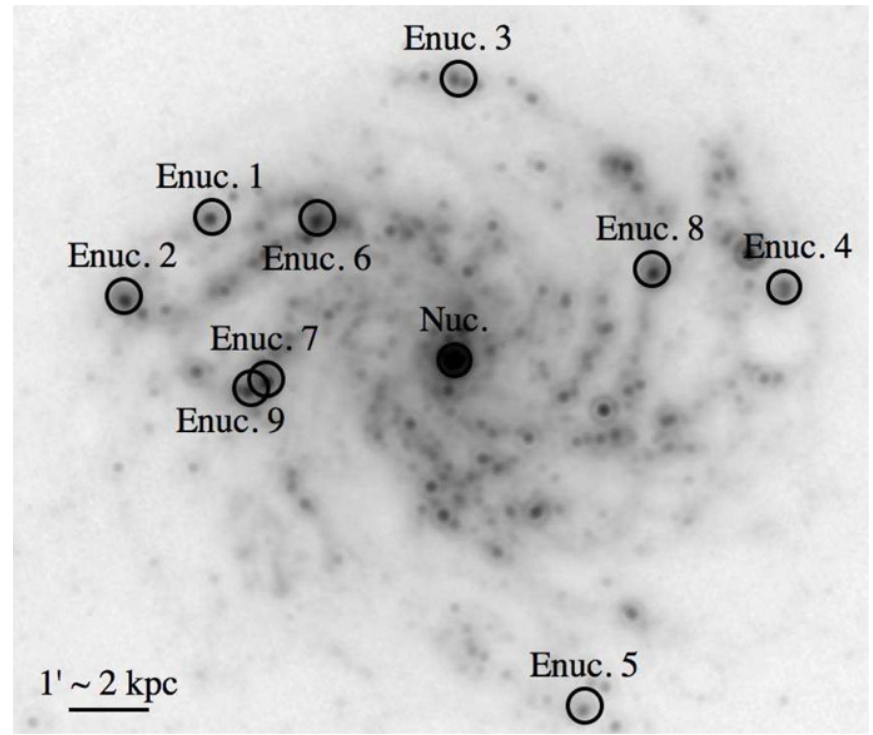

Figure 1. $24 \mu \mathrm{m}$ map of NGC 6946 showing the location of each star-forming region in the present investigation. The diameter of each circle corresponds to $25^{\prime \prime}$, the FWHM of the GBT beam at $33 \mathrm{GHz}$, which sets the resolution of this study. This diameter projects to a linear scale of $0.8 \mathrm{kpc}$ at the distance of NGC 6946.

Murphy et al. (2010). This is done to assess their reliability against SFRs measured via free-free emission at $33 \mathrm{GHz}$. The paper is organized as follows. In Section 2, we introduce the data sets used for our SFR comparisons. Then, in Section 3, we calibrate each of the observed quantities for the same initial mass function (IMF). Comparisons between each of the SFR diagnostics are made in Section 4 and discussed in Section 5. Our main conclusions are summarized in Section 6.

\section{DATA AND PHOTOMETRY}

NGC 6946 is a nearby $(d \approx 6.8 \mathrm{Mpc}$; Karachentsev et al. 2000) late-type spiral galaxy harboring a mild starburst nucleus (Turner \& Ho 1983; Ball et al. 1985). Recently, Murphy et al. (2010) targeted 10 star-forming regions within NGC 6946, including the nucleus, using the $100 \mathrm{~m}$ Robert C. Byrd Green Bank Telescope (GBT) in the Ka band (26-40 GHz). These regions were selected because they have existing and forthcoming mid- and far-infrared spectroscopic data collected as part of the Spitzer Infrared Nearby Galaxies Survey (SINGS; Kennicutt et al. 2003) and the project, Key Insights on Nearby Galaxies: a Far-Infrared Survey with Herschel (KINGFISH; PI: R. C. Kennicutt). The position of each source is given in Table 1. In Figure 1, we show the location of each region on the $24 \mu \mathrm{m}$ image of NGC 6946; the corresponding circle diameters are $25^{\prime \prime}$, which match the FWHM of our lowest resolution data for the present multiwavelength SFR comparison. This projects to a physical scale of $\approx 0.8 \mathrm{kpc}$ at the distance of NGC 6946 .

\subsection{Radio Data}

Radio data at $1.4,1.5,1.7,4.9,8.5$, and $33 \mathrm{GHz}$ used here are the same as the data presented in Murphy et al. (2010), where a more detailed description of the observations and spectral decomposition of the thermal and non-thermal components can be found. The $1.4 \mathrm{GHz}$ radio map $\left(14^{\prime \prime} \times 12^{\prime \prime} .5\right.$ beam $)$ comes from the Westerbork Synthesis Radio Telescope SINGS survey (Braun et al. 2007). Imaging at 1.5, 1.7, 4.9, and $8.5 \mathrm{GHz}$ $\left(15^{\prime \prime} \times 15^{\prime \prime}\right.$ beam) all come from Beck (2007). We note that the 
Table 1

Source Positions and Radio Photometry

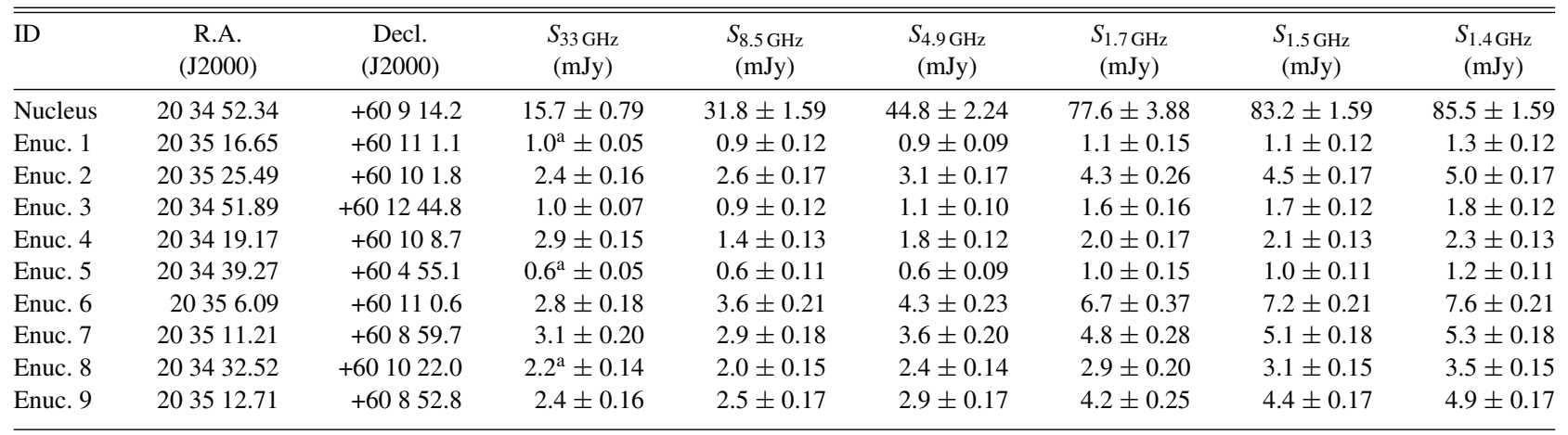

Note. " Correction ("boosting") factors arising from an oversubtraction of signal by reference beams have been applied to 33 GHz flux densities for sources in which the correction was larger than $15 \%$. These scale factors are $2.55,1.17$, and 1.25 for Enuc. 1, 5, and 8 , respectively.

4.9 and $8.5 \mathrm{GHz}$ radio data included single-dish measurements for short-spacing corrections.

Observations at $33 \mathrm{GHz}$ were taken on 2009 March 21 using the Caltech Continuum Backend (CCB) on the GBT. The CCB simultaneously measures the entire $\mathrm{Ka}$ bandwidth over four equally spaced frequency channels. The beamswitched signal is synchronously read out and demodulated to remove atmospheric fluctuation and/or gain variations. Reference beams are measured by nodding 1'.3 away from the source; details about the reference beam locations can be found in Figure 1 of Murphy et al. (2010).

The average FWHM of the GBT beam in the Ka band was $\approx 25^{\prime \prime}$ among our sets of observations. A detailed description on the performance of the $\mathrm{CCB}$ receiver, the data reduction pipeline, and error estimates is given in Mason et al. (2009).

\subsection{UV, Optical, and IR Data}

Galaxy Evolution Explorer (GALEX) far-UV (FUV; $1528 \AA$ ) and near-UV (NUV; $2271 \AA$ ) data of NGC 6946 were taken from the GALEX archive and will be included in the GALEX Large Galaxy Atlas (M. Seibert et al. 2011, in preparation). The calibration uncertainty for these data is $\approx 15 \%$ in both bands. The $\mathrm{H} \alpha$ image of NGC 6946, obtained using the Tek $2048 \times 2048$ CCD on the KPNO $0.9 \mathrm{~m}\left(23^{\prime} \times 23^{\prime}\right.$ field of view $(\mathrm{FOV})$ and 0.69 pixel $^{-1}$ ), was taken from Ferguson et al. (1998) and has a calibration uncertainty of $\approx 20 \%$. The 8,24 , and $850 \mu \mathrm{m}$ data used here were included in the SINGS fifth data release ${ }^{18}$ and have calibration uncertainties of $5 \%, 5 \%$, and $15 \%$, respectively. Regions 2, 3, and 5 were not covered by the SCUBA $850 \mu \mathrm{m}$ maps, while regions 4 and 8 were near the edges of the maps. We note that the $8 \mu \mathrm{m}$ map was corrected for stellar light using a scaled $3.6 \mu \mathrm{m}$ image following Helou et al. (2004). We do not use the Spitzer $70 \mu \mathrm{m}$ or the SCUBA $450 \mu \mathrm{m}$ data, also included in the SINGS fifth data release, in lieu of new Herschel data taken as part of KINGFISH.

We make use of the Herschel (Pilbratt et al. 2010) data obtained by the KINGFISH project having resolutions better than the $25^{\prime \prime}$ beam of our GBT data which include PACS 70 , 100 , and $160 \mu \mathrm{m}$ imaging, along with SPIRE 250 and $350 \mu \mathrm{m}$ imaging. Both PACS and SPIRE imaging were reduced using the Herschel Interactive Processing Environment (Ott 2010) software package, version 3.0.1510. The current calibration

\footnotetext{
${ }^{18}$ SINGS data products can be found at http://irsa.ipac.caltech.edu/data/SPITZER/SINGS/.
}

has uncertainties of $\sim 10 \%, 10 \%$, and $20 \%$ for the 70,100 , and $160 \mu \mathrm{m}$ bands, respectively (Poglitsch et al. 2010). The uncertainty in the SPIRE 250 and $350 \mu \mathrm{m}$ data is taken to be $15 \%$, which is the quadrature sum of the $15 \%$ absolute calibration uncertainty and a $1 \%$ uncertainty in the size of the beam; following the current recommendation by the SPIRE Observer's Manual, we assume beam sizes at 250 and $350 \mu \mathrm{m}$ of 426 and $771 \operatorname{arcsec}^{2}$, respectively. Calibration correction factors of 1.02 and 1.05 were applied to the 250 and $350 \mu \mathrm{m}$ data as suggested in the SPIRE Scan-Map AOT Observer's manual.

\subsection{Photometry}

The photometry was carried out on all UV, optical, infrared, and radio maps after first cropping each image to a common FOV and regridding to a common pixel scale. To accurately match the photometry of our images to the GBT measurements, maps were convolved to the resolution of the GBT beam in the Ka band following the image registration description given in Murphy et al. (2010). Registration of the Herschel data was carried out in a similar way as the registration of the Spitzer data. Each of the Herschel images was first convolved to the resolution of the $350 \mu \mathrm{m}$ data using custom smoothing kernels as described in Gordon et al. (2008) and updated by those authors for Herschel, CLEANed to suppress side-lobe structure in the point spread function, and then restored using a Gaussian beam having an FWHM of 25". Flux densities at each wavelength were measured by taking the surface brightness at the location of each GBT pointing and multiplying by the effective area of the beam. In the case of the UV and $\mathrm{H} \alpha$ photometry, we correct each region for Milky Way extinction using Schlegel et al. (1998) assuming $A_{V} / E(B-V)=3.1$ and the modeled extinction curves of Weingartner \& Draine (2001) and Draine (2003).

Unlike Murphy et al. (2010), we subtract a local background estimate from our photometry within the vicinity of each starforming region to remove any diffuse emission component that is most likely unassociated with ongoing star formation. Results using the photometry without subtracting local backgrounds are discussed in Section 5.4 and in the Appendix. The local background estimates were measured by placing four $25^{\prime \prime}$ diameter apertures at a distance of 1.5 times the beam FWHM (i.e., 37".5) away from the center of the source positions in each of the four cardinal directions. The median surface brightness among the pixels within these four apertures (typically 100 pixels in total) was then multiplied by the effective area of the beam to get an 
Table 2

Infrared Photometry

\begin{tabular}{|c|c|c|c|c|c|c|c|c|}
\hline ID & $\begin{array}{c}f_{v}(8 \mu \mathrm{m})^{\mathrm{a}} \\
(\mathrm{mJy})\end{array}$ & $\begin{array}{c}f_{v}(24 \mu \mathrm{m}) \\
(\mathrm{mJy})\end{array}$ & $\begin{array}{c}f_{v}(70 \mu \mathrm{m}) \\
(\mathrm{Jy})\end{array}$ & $\begin{array}{c}f_{v}(100 \mu \mathrm{m}) \\
(\mathrm{Jy})\end{array}$ & $\begin{array}{c}f_{v}(160 \mu \mathrm{m}) \\
(\mathrm{Jy})\end{array}$ & $\begin{array}{c}f_{v}(250 \mu \mathrm{m}) \\
(\mathrm{Jy})\end{array}$ & $\begin{array}{c}f_{v}(350 \mu \mathrm{m}) \\
(\mathrm{Jy})\end{array}$ & $\begin{array}{c}f_{\nu}(850 \mu \mathrm{m}) \\
(\mathrm{mJy})\end{array}$ \\
\hline Nucleus & $1506.7 \pm 75.4$ & $4308.9 \pm 215.4$ & $26.11 \pm 2.61$ & $32.09 \pm 3.21$ & $23.67 \pm 4.74$ & $6.80 \pm 1.09$ & $3.12 \pm 0.50$ & $401.8 \pm 62.8$ \\
\hline Enuc. 1 & $33.6 \pm 2.8$ & $88.0 \pm 4.5$ & $0.85 \pm 0.13$ & $1.23 \pm 0.18$ & $1.02 \pm 0.23$ & $0.33 \pm 0.06$ & $0.15 \pm 0.04$ & $10.8 \pm 17.9$ \\
\hline Enuc. 2 & $53.0 \pm 3.5$ & $175.1 \pm 8.8$ & $1.28 \pm 0.16$ & $1.94 \pm 0.24$ & $1.65 \pm 0.34$ & $0.48 \pm 0.08$ & $0.22 \pm 0.05$ & $\cdots$ \\
\hline Enuc. 3 & $40.6 \pm 3.1$ & $66.4 \pm 3.5$ & $1.00 \pm 0.14$ & $1.54 \pm 0.21$ & $1.30 \pm 0.28$ & $0.36 \pm 0.07$ & $0.20 \pm 0.05$ & $\ldots$ \\
\hline Enuc. 4 & $51.9 \pm 3.5$ & $83.1 \pm 4.3$ & $1.00 \pm 0.14$ & $1.65 \pm 0.21$ & $1.49 \pm 0.32$ & $0.35 \pm 0.07$ & $0.17 \pm 0.04$ & $15.2 \pm 17.9$ \\
\hline Enuc. 6 & $192.3 \pm 9.9$ & $352.2 \pm 17.6$ & $2.62 \pm 0.28$ & $3.77 \pm 0.40$ & $3.02 \pm 0.61$ & $0.69 \pm 0.12$ & $0.37 \pm 0.07$ & $54.7 \pm 19.6$ \\
\hline Enuc. 7 & $186.6 \pm 9.6$ & $366.0 \pm 18.3$ & $3.21 \pm 0.34$ & $4.37 \pm 0.46$ & $3.65 \pm 0.74$ & $1.21 \pm 0.20$ & $0.57 \pm 0.10$ & $60.5 \pm 20.0$ \\
\hline Enuc. 8 & $114.7 \pm 6.2$ & $178.5 \pm 9.0$ & $1.55 \pm 0.19$ & $2.27 \pm 0.26$ & $1.92 \pm 0.40$ & $0.56 \pm 0.10$ & $0.26 \pm 0.05$ & $39.6 \pm 18.8$ \\
\hline Enuc. 9 & $170.3 \pm 8.8$ & $301.2 \pm 15.1$ & $2.25 \pm 0.25$ & $3.31 \pm 0.36$ & $3.03 \pm 0.61$ & $0.95 \pm 0.16$ & $0.46 \pm 0.08$ & $58.8 \pm 19.9$ \\
\hline
\end{tabular}

Note. ${ }^{\text {a }}$ Corrected for stellar emission. Observations were made using the following observatories and instruments: Spitzer-IRAC ( $4 \mu \mathrm{m}$ ), Spitzer-MIPS $(24 \mu \mathrm{m})$, Herschel-PACS $(70,100,160 \mu \mathrm{m})$, Herschel-SPIRE $(250,350 \mu \mathrm{m})$, and JCMT-SCUBA $(850 \mu \mathrm{m})$.

estimate of the local diffuse background emission. While such a local background subtraction for our $33 \mathrm{GHz}$ GBT singlepointing observations is not possible, we note that those observations were taken using 1.3 nods, thus reference beams were typically measured on diffuse parts of the galaxy and likely provide a reasonable measure of the local background. Furthermore, at $33 \mathrm{GHz}$, we do not expect there to be a significant underlying diffuse component if the emission is in fact dominated by free-free emission.

Photometric uncertainties were conservatively estimated by taking the quadrature sum of the calibration and background uncertainties, along with the rms noise for each image. The results from our radio, infrared, and $\mathrm{UV} / \mathrm{H} \alpha$ photometry, along with $1 \sigma$ uncertainties, are given in Tables $1-3$, respectively. The local background values, along with the average fractional contribution of the background emission at each waveband and region, are given in the Appendix. For the cases in which an oversubtraction of signal in the $33 \mathrm{GHz}$ observations has been estimated to be at the $\gtrsim 15 \%$ level due to reference beams landing on bright regions in the galaxy disk, we apply correction ("boosting") factors to the $33 \mathrm{GHz}$ flux densities (see Murphy et al. 2010). These scale factors were derived based on the $8.5 \mathrm{GHz}$ and $24 \mu \mathrm{m}$ images and are approximately $2.55,1.17$, and 1.25 for Enuc. 1, 5, and 8, respectively. We note that these values are $\lesssim 2 \%$ smaller than those reported by Murphy et al. (2010) due to including local background subtractions.

\subsection{Modeling the Infrared to Radio Spectra}

We fit the radio and infrared data independently given that the physical processes producing these emissions are distinct. The infrared (i.e., 8-850 $\mu \mathrm{m}$ ) photometry is fit by the spectral energy distribution (SED) models of Dale \& Helou (2002); associated total infrared (IR: 8-1000 $\mu \mathrm{m}$ ) luminosities from integrating the best-fit SEDs for each region are given in Table 4. Fitting errors in the IR luminosity determinations were estimated by a standard Monte Carlo approach using the photometric uncertainties of the input flux densities.

The radio data are fit by varying a combination of thermal (free-free) and non-thermal (synchrotron) emission components which scale as $S_{v}^{\mathrm{T}} \propto v^{-\alpha_{\mathrm{T}}}$ and $S_{v}^{\mathrm{NT}} \propto v^{-\alpha_{\mathrm{NT}}}$, respectively, where $\alpha_{\mathrm{T}}=0.1$ and $\alpha_{\mathrm{NT}}$ (see Table 4 for estimated values) are the thermal and non-thermal spectral indices, respectively. The only exception to this is for extranuclear region 4 where the thermal and non-thermal emission were fit only to the radio
Table 3

$\mathrm{H} \alpha$ and UV Photometry

\begin{tabular}{lcccc}
\hline \hline ID & $\begin{array}{c}f_{\mathrm{H} \alpha}{ }^{\mathrm{a}} \\
\left(10^{-12} \mathrm{erg} \mathrm{s}^{-1} \mathrm{~cm}^{-2}\right)\end{array}$ & $\begin{array}{c}f_{\nu}(1528 \AA) \\
(\mathrm{mJy})\end{array}$ & $\begin{array}{c}f_{v}(2271 \AA) \\
(\mathrm{mJy})\end{array}$ & $\begin{array}{c}E(B-V)^{\mathrm{b}} \\
(\mathrm{mag})\end{array}$ \\
\hline Nucleus & $1.09 \pm 0.22$ & $0.07 \pm 0.01$ & $0.42 \pm 0.06$ & 0.342 \\
Enuc. 1 & $0.47 \pm 0.09$ & $1.25 \pm 0.19$ & $2.49 \pm 0.37$ & 0.345 \\
Enuc. 2 & $2.01 \pm 0.40$ & $5.81 \pm 0.87$ & $9.58 \pm 1.44$ & 0.346 \\
Enuc. 3 & $0.80 \pm 0.16$ & $2.52 \pm 0.38$ & $4.44 \pm 0.67$ & 0.343 \\
Enuc. 4 & $0.61 \pm 0.12$ & $1.65 \pm 0.25$ & $3.30 \pm 0.50$ & 0.343 \\
Enuc. 5 & $0.45 \pm 0.09$ & $1.04 \pm 0.16$ & $2.06 \pm 0.31$ & 0.335 \\
Enuc. 6 & $1.09 \pm 0.22$ & $1.80 \pm 0.27$ & $3.75 \pm 0.56$ & 0.344 \\
Enuc. 7 & $1.19 \pm 0.24$ & $1.93 \pm 0.29$ & $4.07 \pm 0.61$ & 0.342 \\
Enuc. 8 & $0.36 \pm 0.07$ & $0.23 \pm 0.04$ & $0.67 \pm 0.10$ & 0.342 \\
Enuc. 9 & $0.76 \pm 0.15$ & $1.16 \pm 0.17$ & $2.44 \pm 0.37$ & 0.342 \\
\hline
\end{tabular}

Notes.

${ }^{\text {a }} \mathrm{H} \alpha$ imaging obtained with the KPNO $0.9 \mathrm{~m}$ by Ferguson et al. (1998).

${ }^{\mathrm{b}}$ Galactic extinction taken from Schlegel et al. (1998) used to correct the $\mathrm{H} \alpha$ and GALEX FUV and NUV flux densities assuming $A_{V} / E(B-V)=3.1$ and the modeled extinction curves of Weingartner \& Draine (2001) and Draine (2003).

data at $v<10 \mathrm{GHz}$ and an additional component (i.e., spinning dust emission; Ali-Haïmoud et al. 2009) was used to fit the excess $33 \mathrm{GHz}$ emission (see Murphy et al. 2010 for details). Recent follow-up observations of this region at $16.5 \mathrm{GHz}$ using the Arcminute Microkelvin Imager have confirmed a rising spectrum between 8.5 and $15 \mathrm{GHz}$ (Scaife et al. 2010). The excess emission accounts for $\approx 56 \%$ of the total $33 \mathrm{GHz}$ flux density from extranuclear region 4 and has been subtracted out for the present analysis. We note that the quantitative results presented here are significantly different from what is given in Murphy et al. (2010) as those authors did not subtract out a local background estimate and included a spinning dust component for star-forming regions in addition to extranuclear region 4. Results associated with fitting the photometry without subtracting a local background can be found in Section 5.4 and in the Appendix.

Before varying the free-free and non-thermal components to fit the observed radio spectra, we first estimate the non-thermal spectral index for each source. The non-thermal emission is proportional to the ratio of energy losses of CR electrons from synchrotron emission to total energy losses. In our model, we account for energy losses to $\mathrm{CR}$ electrons arising from synchrotron radiation, inverse Compton (IC) scattering, ionization, bremsstrahlung, and escape through an empirical prescription. Additional losses through advection from a galactic scale wind 
Table 4

Derived Parameters

\begin{tabular}{|c|c|c|c|c|c|c|}
\hline ID & $\begin{array}{c}f_{\mathrm{T}}^{33 \mathrm{GHz}} \\
(\%)\end{array}$ & $\alpha^{\text {obsa }}$ & $\alpha^{\mathrm{NTa}}$ & $\begin{array}{c}L_{\mathrm{IR}} \\
\left(10^{8} L_{\odot}\right)\end{array}$ & $\begin{array}{l}B_{\min }{ }^{\mathrm{b}} \\
(\mu \mathrm{G})\end{array}$ & $\begin{array}{c}T_{\mathrm{e}} \\
\left(10^{4} \mathrm{~K}\right)\end{array}$ \\
\hline Nucleus & $62 \pm 6$ & $0.58 \pm 0.04$ & $0.74 \pm 0.05$ & $47.56 \pm 2.14$ & $30.3 \pm 9.3$ & $0.42 \pm 0.08$ \\
\hline Enuc. 1 & $93 \pm 4$ & $0.10 \pm 0.01$ & $0.78 \pm 0.06$ & $1.08 \pm 0.08$ & $10.1 \pm 3.0$ & $1.36 \pm 0.31$ \\
\hline Enuc. 2 & $87 \pm 5$ & $0.29 \pm 0.02$ & $0.82 \pm 0.06$ & $1.92 \pm 0.11$ & $14.3 \pm 4.1$ & $0.77 \pm 0.27$ \\
\hline Enuc. 3 & $87 \pm 7$ & $0.23 \pm 0.02$ & $0.79 \pm 0.06$ & $1.10 \pm 0.08$ & $11.0 \pm 3.2$ & $0.86 \pm 0.31$ \\
\hline Enuc. 4 & $42^{c} \pm 4$ & $0.20 \pm 0.01$ & $0.80 \pm 0.06$ & $1.32 \pm 0.08$ & $11.6 \pm 3.4$ & $1.78 \pm 0.59$ \\
\hline Enuc. 5 & $89 \pm 8$ & $0.24 \pm 0.02$ & $0.77 \pm 0.05$ & $0.41 \pm 0.05$ & $9.9 \pm 2.9$ & $1.07 \pm 0.44$ \\
\hline Enuc. 6 & $86 \pm 5$ & $0.38 \pm 0.03$ & $0.83 \pm 0.06$ & $4.72 \pm 0.25$ & $16.0 \pm 4.6$ & $1.04 \pm 0.23$ \\
\hline Enuc. 7 & $85 \pm 5$ & $0.23 \pm 0.02$ & $0.83 \pm 0.06$ & $4.86 \pm 0.26$ & $14.5 \pm 4.2$ & $1.14 \pm 0.25$ \\
\hline Enuc. 8 & $88 \pm 5$ & $0.18 \pm 0.01$ & $0.81 \pm 0.06$ & $2.66 \pm 0.14$ & $13.1 \pm 3.8$ & $2.80 \pm 0.54$ \\
\hline Enuc. 9 & $85 \pm 5$ & $0.28 \pm 0.02$ & $0.83 \pm 0.06$ & $4.12 \pm 0.21$ & $14.3 \pm 4.1$ & $1.22 \pm 0.26$ \\
\hline
\end{tabular}

Notes.

${ }^{a}$ The observed and non-thermal radio spectral indices measured between 1.4 and $8.5 \mathrm{GHz}$.

${ }^{\mathrm{b}}$ Calculated using the total $1.4 \mathrm{GHz}$ flux densities and the revised minimum energy calculation of Beck \& Krause (2005).

${ }^{c}$ Calculated using the observed $33 \mathrm{GHz}$ flux density before subtracting out the $\approx 56 \%$ contribution thought to arise from anomalous dust emission (see Section 2.4).

are ignored. A much more detailed description of the construction of the modeled radio spectra can be found in Murphy (2009).

Synchrotron losses are proportional to $U_{B}=B^{2} /(8 \pi)$, the magnetic field energy density, while IC losses are proportional to $U_{\text {rad }}$, the radiation field energy density. We use magnetic field strengths estimated from the $1.4 \mathrm{GHz}$ radio continuum surface brightnesses following the revised minimum energy calculation of Beck \& Krause (2005; see Table 4). For this calculation, we assume a proton-to-electron number density ratio of $K_{0} \approx 100 \pm 50$ and a path length of $l \approx 1 \pm 0.5 \mathrm{kpc}$. The estimated $B_{\min }$ values range between $\approx 10$ and $16 \mu \mathrm{G}$ among the extranuclear star-forming regions and it is $\approx 30 \mu \mathrm{G}$ for the nucleus. Assuming that the bolometric surface brightness of these dusty star-forming regions is well approximated by the IR surface brightness, we estimate $U_{\text {rad }} \approx(2 \pi / c) I_{\mathrm{IR}}$. These values range between $\approx(0.4-4.2) \times 10^{-12} \mathrm{erg} \mathrm{cm}^{-3}$ among the extranuclear star-forming regions, and it is $\approx 41 \times 10^{-12} \mathrm{erg} \mathrm{cm}^{-3}$ in the nucleus. We assume that the density of the interstellar medium (ISM) is $n_{\mathrm{ISM}}=0.1$ and $10 \mathrm{~cm}^{-3}$ for the extranuclear regions and nucleus, respectively. Taking these values, we make a rough estimate for the shape (i.e., spectral index) of the nonthermal radio spectrum. Once the non-thermal spectral index is fixed, the thermal and non-thermal components are varied to fit the radio observations.

The radio to infrared spectra of each star-forming region are plotted in Figure 2, along with the components of the fits. In Table 4, we list the observed ( $\left.\alpha^{\mathrm{obs}}\right)$ and (estimated) non-thermal radio spectral indices measured between 1.4 and $8.5 \mathrm{GHz}$, along with the associated thermal radio fractions at $33 \mathrm{GHz}$ from our radio spectral fitting for each star-forming region. The observed indices are quite flat, being $\approx 0.2$, on average, consistent with young $\mathrm{H}$ II regions, while the average non-thermal spectral index is $\approx 0.8$. The average $33 \mathrm{GHz}$ thermal fraction is found to be $\approx 87 \%$ among these star-forming regions.

\section{SFR CALIBRATIONS}

To ensure that each SFR diagnostic can be compared fairly, we derive corresponding calibrations using Starburst99 (Leitherer et al. 1999) for a common IMF. We choose a Kroupa (Kroupa 2001) IMF, having a slope of -1.3 for stellar masses between $0.1-0.5 M_{\odot}$ and -2.3 for stellar masses ranging between 0.5 and $100 M_{\odot}$. These calibrations update those presented

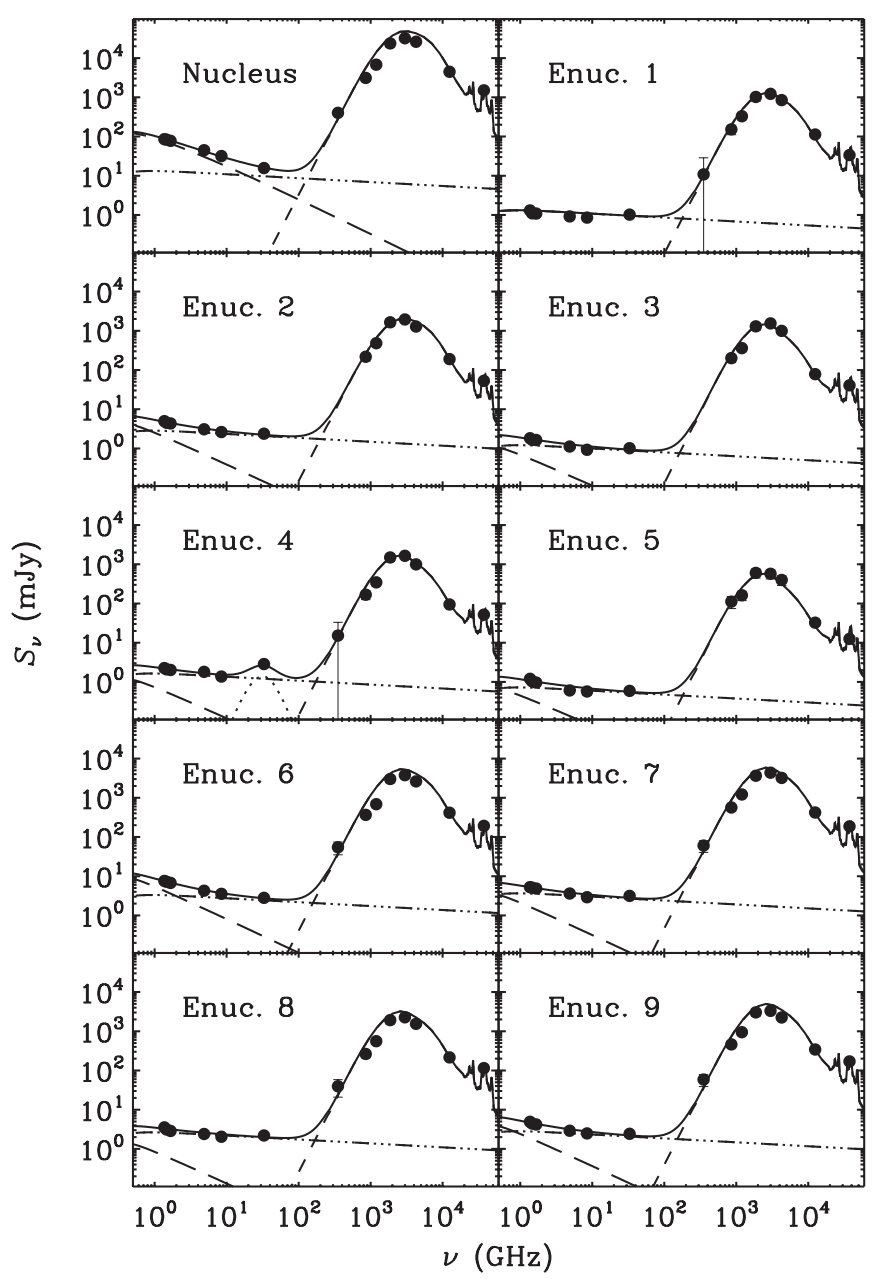

Figure 2. Radio to infrared spectra of each star-forming region. The radio and infrared photometry are plotted as filled circles; error bars are shown, but are usually smaller than the size of the plotting symbol. Fits to the data using a combination of modeled radio spectra (Murphy 2009) and infrared SED libraries of Dale \& Helou (2002) are shown as solid lines. The infrared dust emission is shown by a short-dashed line. The radio spectrum is modeled by two components, non-thermal synchrotron emission (long-dashed lines) and thermal (free-free) emission (triple-dot-dashed line) except for the case of extranuclear region 4, where an additional component (i.e., spinning dust emission; Ali-Haïmoud et al. 2009, dotted line) was needed to fit the excess emission at $33 \mathrm{GHz}$ (see Murphy et al. 2010 for details). 
in Kennicutt (1998). Only SFR diagnostics that are considered to be reliable independent of extinction are discussed.

\subsection{UV, Optical, and IR SFRs}

Assuming a solar metallicity and continuous star formation (i.e., a fixed SFR), Starburst99 stellar population models yield the following relation between the SFR and production rate of ionizing photons, $Q\left(H^{0}\right)$, at an age of $\sim 100 \mathrm{Myr}$ :

$$
\left(\frac{\mathrm{SFR}}{M_{\odot} \mathrm{yr}^{-1}}\right)=7.29 \times 10^{-54}\left[\frac{Q\left(H^{0}\right)}{\mathrm{s}^{-1}}\right] .
$$

For reference, the coefficient relating the SFR and ionizing photon rate given in Kennicutt (1998) is a factor of $\approx 1.5$ times larger than that above (see Calzetti et al. 2007 for a discussion on the origin of this difference). We choose a constant SFR over a timescale of $\sim 100 \mathrm{Myr}$ for all estimators as we are sampling star-forming complexes on the scales of $\approx 0.8 \mathrm{kpc}$ that likely include a range of ages among individual star formation sites. Consequently, below certain spatial scales (i.e., $\lesssim$ a few hundred parsecs; the size of individual giant $\mathrm{H}$ II regions) these calibrations will likely break down as the assumption of spatial averaging is no longer valid.

The ionizing photon rate can of course be expressed as an (extinction corrected) $\mathrm{H}$ recombination line flux, such that for Case $\mathrm{B}$ recombination, and assuming an electron temperature $T_{\mathrm{e}}=10^{4} \mathrm{~K}$, the $\mathrm{H} \alpha$ recombination line strength is related to the SFR by

$$
\left(\frac{\mathrm{SFR}_{\mathrm{H} \alpha}}{M_{\odot} \mathrm{yr}^{-1}}\right)=5.37 \times 10^{-42}\left(\frac{L_{\mathrm{H} \alpha}}{\mathrm{erg} \mathrm{s}^{-1}}\right) .
$$

The above equation indicates that the SFR is directly proportional to the $\mathrm{H} \alpha$ line luminosity, assuming that a constant fraction of the ionized $\mathrm{H}$ atoms will emit an $\mathrm{H} \alpha$ photon as they recombine and that the extinction correction is accurate. However, if a significant fraction of ionizing photons are absorbed by dust, the above equation will underestimate the SFR, and the = sign should be replaced by $\geqslant$. Since the ionizing flux comes from very massive stars with lifetimes $\lesssim 10 \mathrm{Myr}$, we note that the coefficients in Equations (1) and (2) are nearly independent of starburst age under the assumption of continuous star formation so long at it is $\gtrsim 10 \mathrm{Myr}$. Accordingly, such measurements sample the current (i.e., $10 \mathrm{Myr}$ ) star formation activity.

The integrated UV spectrum is dominated by young stars, making it a sensitive probe of recent $(\sim 10-100$ Myr; Kennicutt 1998; Calzetti et al. 2005; Salim et al. 2007) star formation activity. We convolve the output Starburst99 spectrum with the GALEX FUV transmission curve to obtain the following conversion between SFR and FUV luminosity,

$$
\left(\frac{\mathrm{SFR}_{\mathrm{FUV}}}{M_{\odot} \mathrm{yr}^{-1}}\right)=4.42 \times 10^{-44}\left(\frac{L_{\mathrm{FUV}}}{\mathrm{erg} \mathrm{s}^{-1}}\right) .
$$

To derive a calibration for the total infrared (IR; 8-1000 $\mu \mathrm{m}$ ), we make the assumption that the entire Balmer continuum is absorbed and re-radiated by dust and that the dust emission is optically thin. Integrating the output Starburst99 spectrum over this wavelength range (i.e., $912 \AA<\lambda<3646 \AA$ ) results in the following (predicted) relation between the IR emission and current SFR:

$$
\left(\frac{\mathrm{SFR}_{\mathrm{IR}}}{M_{\odot} \mathrm{yr}^{-1}}\right)=3.88 \times 10^{-44}\left(\frac{L_{\mathrm{IR}}}{\mathrm{erg} \mathrm{s}^{-1}}\right) .
$$

Since a non-negligible fraction of the far-infrared continuum may be heated by an older stellar population (e.g., Sauvage \& Thuan 1992; Walterbos \& Greenawalt 1996; Bendo et al. 2010), it is likely more physical to only relate warmer dust emission to the current SFR (e.g., Helou et al. 2004). Thus, we also include a monochromatic $24 \mu \mathrm{m}$ based SFR diagnostic, for which there are a number of calibrations in the literature (e.g., Wu et al. 2005; Pérez-González et al. 2006; Alonso-Herrero et al. 2006; Calzetti et al. 2007; Relaño et al. 2007; Zhu et al. 2008; Rieke et al. 2009). A detailed comparison among each of these relations can be found in Calzetti et al. (2010). For simplicity, we only choose a single calibration to use in our comparison (i.e., Relaño et al. 2007; calibrated for a Kroupa IMF), such that

$$
\left(\frac{\mathrm{SFR}_{24} \mu \mathrm{m}}{M_{\odot} \mathrm{yr}^{-1}}\right)=5.58 \times 10^{-36}\left[\frac{\nu L_{v}(24 \mu \mathrm{m})}{\mathrm{erg} \mathrm{s}^{-1}}\right]^{0.826},
$$

as this relation has been calibrated over the largest range in $24 \mu \mathrm{m}$ luminosity (i.e., $1 \times 10^{38} \mathrm{erg} \mathrm{s}^{-1} \lesssim \nu L_{\nu}(24 \mu \mathrm{m}) \lesssim$ $3 \times 10^{44} \mathrm{erg} \mathrm{s}^{-1}$ ).

Given that not all of the UV/optical photons will be absorbed and re-radiated by dust, a series of new empirical calibrations based on the linear combination of observed $24 \mu \mathrm{m}$ (obscured star formation) and $\mathrm{H} \alpha$ (unobscured star formation) luminosities have been developed (e.g., Calzetti et al. 2007; Kennicutt et al. 2007, 2009; Zhu et al. 2008). Based on the analysis of Calzetti et al. (2007), we adopt the following relation for our sample of star-forming regions to obtain extinction-corrected $\mathrm{H} \alpha$ line luminosities:

$$
\left(\frac{L_{\mathrm{H} \alpha}^{\mathrm{corr}}}{\mathrm{erg} \mathrm{s}^{-1}}\right)=\left[\frac{L_{\mathrm{H} \alpha}^{\mathrm{obs}}+0.031 \nu L_{\nu}(24 \mu \mathrm{m})}{\operatorname{erg~s}^{-1}}\right] .
$$

While this relation has been calibrated for $\mathrm{H}$ II regions, we note that it has also been found to be appropriate for entire galaxies having $4 \times 10^{42} \leqslant v L_{v}(24 \mu \mathrm{m})<5 \times 10^{43} \mathrm{erg} \mathrm{s}^{-1}$. For galaxies having $\nu L_{v}(24 \mu \mathrm{m})<4 \times 10^{42} \mathrm{erg} \mathrm{s}^{-1}$, this relation is optimized by replacing the coefficient 0.031 by 0.020 (Calzetti et al. 2010), which is the calibration derived for normal star-forming galaxies by Kennicutt et al. (2009). Combining Equation (2) with Equation (6) results in the following SFR relation:

$$
\left(\frac{\mathrm{SFR}_{\text {mix }}}{M_{\odot} \mathrm{yr}^{-1}}\right)=5.37 \times 10^{-42}\left[\frac{L_{\mathrm{H} \alpha}^{\mathrm{obs}}+0.031 \nu L_{v}(24 \mu \mathrm{m})}{\mathrm{erg} \mathrm{s}^{-1}}\right] .
$$

Similarly, by combining the SFR estimates from the total IR and (observed) UV emission, one can account for the obscured and unobscured emission contributing to the total (bolometric) SFR, which we define as

$$
\mathrm{SFR}_{\mathrm{tot}}=\mathrm{SFR}_{\mathrm{FUV}}+\mathrm{SFR}_{\mathrm{IR}}
$$

Using the above calibrations, this relation can be expressed as a linear combination of the UV and total IR emission such that

$$
\left(\frac{\mathrm{SFR}_{\mathrm{tot}}}{M_{\odot} \mathrm{yr}^{-1}}\right)=4.42 \times 10^{-44}\left(\frac{L_{\mathrm{FUV}}+0.88 L_{\mathrm{IR}}}{\mathrm{erg} \mathrm{s}^{-1}}\right) .
$$

This diagnostic is often used to characterize SFRs from galaxies in both low- (e.g., Iglesias-Páramo et al. 2006; Buat et al. 2007, 2011) and high-z (e.g., Iglesias-Páramo et al. 2004; Elbaz et al. 2007; Daddi et al. 2007; Reddy et al. 2010) studies. However, as already mentioned above, the presented calibrations will depend 
on each complex including a range of ages among individual star formation sites. Therefore, calibrations such as these will have increasing uncertainties when applied to a single star-forming region depending on how well the IR and UV are measuring emission from recent star formation, not to mention the fact that the IR and UV are sensitive to star formation on different timescales (i.e., $\sim 10 \mathrm{Myr}$ versus $\sim 10-100 \mathrm{Myr}$, respectively). It is also worth noting that the derived coefficient for scaling the IR luminosity given above is larger than that from empirical studies of starburst galaxies which report a coefficient closer to 0.6 (e.g., Meurer et al. 1999; Calzetti 2001).

\subsection{Radio SFRs}

Radio continuum emission from galaxies is generally composed of two optically thin components: non-thermal synchrotron emission associated with CR electrons accelerated in a galaxy's magnetic field, and thermal bremsstrahlung (free-free) emission around massive star-forming regions. The origin of each of these components lies in the process of massive star formation.

At high radio frequencies, where $\tau \ll 1$, the ionizing photon rate is directly proportional to the thermal spectral luminosity, $L_{\nu}^{\mathrm{T}}$, varying only weakly with electron temperature $T_{\mathrm{e}}$ (Rubin 1968), such that

$$
\begin{aligned}
{\left[\frac{Q\left(H^{0}\right)}{\mathrm{s}^{-1}}\right]=} & 6.3 \times 10^{25}\left(\frac{T_{\mathrm{e}}}{10^{4} \mathrm{~K}}\right)^{-0.45}\left(\frac{v}{\mathrm{GHz}}\right)^{0.1} \\
& \times\left(\frac{L_{v}^{\mathrm{T}}}{\operatorname{erg~s}^{-1} \mathrm{~Hz}^{-1}}\right) .
\end{aligned}
$$

As with the $\mathrm{H}$ recombination line fluxes, it is again worth noting that $Q\left(H^{0}\right)$, and consequently the SFR, may in fact be underestimated by the free-free emission if a significant fraction of ionizing photons are absorbed by dust; in this case the = sign in the above equation should be replaced by $\geqslant$. By combining Equations (1) and (10), one can derive a relation between the SFR and thermal radio emission:

$$
\begin{aligned}
\left(\frac{\mathrm{SFR}_{v}^{\mathrm{T}}}{M_{\odot} \mathrm{yr}^{-1}}\right)= & 4.6 \times 10^{-28}\left(\frac{T_{\mathrm{e}}}{10^{4} \mathrm{~K}}\right)^{-0.45}\left(\frac{v}{\mathrm{GHz}}\right)^{0.1} \\
& \times\left(\frac{L_{v}^{\mathrm{T}}}{\mathrm{erg} \mathrm{s}^{-1} \mathrm{~Hz}^{-1}}\right) .
\end{aligned}
$$

Using a Kroupa IMF that extends down to $0.1 M_{\odot}$ results in a coefficient that is a factor of $\sim 2.5$ times larger than that found in the relation between the SFR and free-free radio emission given in Condon (1992), and $\sim 25 \%$ smaller than that given in Schmitt et al. (2006).

At lower radio frequencies, which are typically dominated by non-thermal synchrotron emission, calibrations between the supernova rate, and thus the SFR, have been developed. From the output of Starburst99, which assumed a supernova cutoff mass of $8 M_{\odot}$, we find that the total core-collapse supernova rate, $\dot{N}_{\mathrm{SN}}$, is related to the SFR by

$$
\left(\frac{\mathrm{SFR}}{M_{\odot} \mathrm{yr}^{-1}}\right)=86.3\left(\frac{\dot{N}_{\mathrm{SN}}}{\mathrm{yr}^{-1}}\right) .
$$

Work comparing the non-thermal spectral luminosity with the supernova rate in the Galaxy has yielded an empirical calibration such that

$$
\left(\frac{L_{v}^{\mathrm{NT}}}{\mathrm{erg} \mathrm{s}^{-1} \mathrm{~Hz}^{-1}}\right)=1.3 \times 10^{30}\left(\frac{\dot{N}_{\mathrm{SN}}}{\mathrm{yr}^{-1}}\right)\left(\frac{v}{\mathrm{GHz}}\right)^{-\alpha^{\mathrm{NT}}}
$$

(Tammann 1982; Condon \& Yin 1990). By combining Equations (12) and (13), we can express the SFR as a function of the non-thermal radio emission where

$$
\left(\frac{\mathrm{SFR}_{v}^{\mathrm{NT}}}{M_{\odot} \mathrm{yr}^{-1}}\right)=6.64 \times 10^{-29}\left(\frac{v}{\mathrm{GHz}}\right)^{\alpha^{\mathrm{NT}}}\left(\frac{L_{v}^{\mathrm{NT}}}{\mathrm{erg} \mathrm{s}^{-1} \mathrm{~Hz}^{-1}}\right)
$$

We note that using the Kroupa IMF which extends down to $0.1 M_{\odot}$ results in a coefficient that is a factor of $\sim 3.5$ times larger than that used in the expression relating the SFR and nonthermal radio emission by Condon (1992), and $~ 50 \%$ smaller than that given in Schmitt et al. (2006).

Since the observed radio continuum emission is comprised of both free-free and synchrotron emission, we can combine Equations (11) and (14) to construct a single expression for the SFR from the total radio continuum emission at a given frequency such that

$$
\begin{aligned}
\left(\frac{\mathrm{SFR}_{v}}{M_{\odot} \mathrm{yr}^{-1}}\right)= & 10^{-27}\left[2.18\left(\frac{T_{\mathrm{e}}}{10^{4} \mathrm{~K}}\right)^{0.45}\left(\frac{v}{\mathrm{GHz}}\right)^{-0.1}+15.1\right. \\
& \left.\times\left(\frac{v}{\mathrm{GHz}}\right)^{-\alpha^{\mathrm{NT}}}\right]^{-1}\left(\frac{L_{v}}{\mathrm{erg} \mathrm{s}^{-1} \mathrm{~Hz}^{-1}}\right)
\end{aligned}
$$

This equation essentially weights the observed radio continuum luminosity based on the expected thermal fraction at a given frequency.

The most widely used radio SFR calibration, however, is the result of the tight, empirical FIR-radio correlation (de Jong et al. 1985; Helou et al. 1985):

$$
q_{\mathrm{IR}} \equiv \log \left(\frac{L_{\mathrm{IR}}}{3.75 \times 10^{12} L_{1.4 \mathrm{GHz}}}\right) .
$$

While classically expressed using the integrated (IRAS-based) FIR luminosity, we use the total IR luminosity resulting in an average value of $q_{\mathrm{IR}}=2.64 \pm 0.26 \mathrm{dex}$ for a sample of starforming galaxies in the local universe (Bell 2003); this value is 0.30 dex larger than the average value of $2.34 \pm 0.26$ dex found when using the FIR luminosity (Yun et al. 2001). Thus, one can express the $1.4 \mathrm{GHz}$ luminosity as an SFR by combining Equation (16) with Equation (4):

$$
\left(\frac{\mathrm{SFR}_{1.4 \mathrm{GHz}}}{M_{\odot} \mathrm{yr}^{-1}}\right)=6.35 \times 10^{-29}\left(\frac{L_{1.4 \mathrm{GHz}}}{\mathrm{erg} \mathrm{s}^{-1} \mathrm{~Hz}^{-1}}\right) .
$$

It is worth stating here that this calibration is based on the FIR-radio correlation, which was established for globally integrated FIR and radio continuum properties of galaxies, and thus applies to galaxies as a whole. Most of the non-thermal radio emission from galaxies arises from electrons that have diffused away from their acceleration sites in supernova remnants into quiescent regions void of current star formation. In fact, within individual $\mathrm{H}$ II regions, there may be little non-thermal emission present (e.g., Condon \& Yin 1990). Thus, near star-forming complexes, we expect the non-thermal emission per unit star formation to be low given such a calibration as suggested by studies of the local FIR-radio correlation (e.g., Murphy et al. 2006a; Hughes et al. 2006; Tabatabaei et al. 2007).

\section{COMPARISON OF SFR DIAGNOSTICS}

In the following section, we compare SFRs estimated via the various diagnostics described in Section 3. As previously 


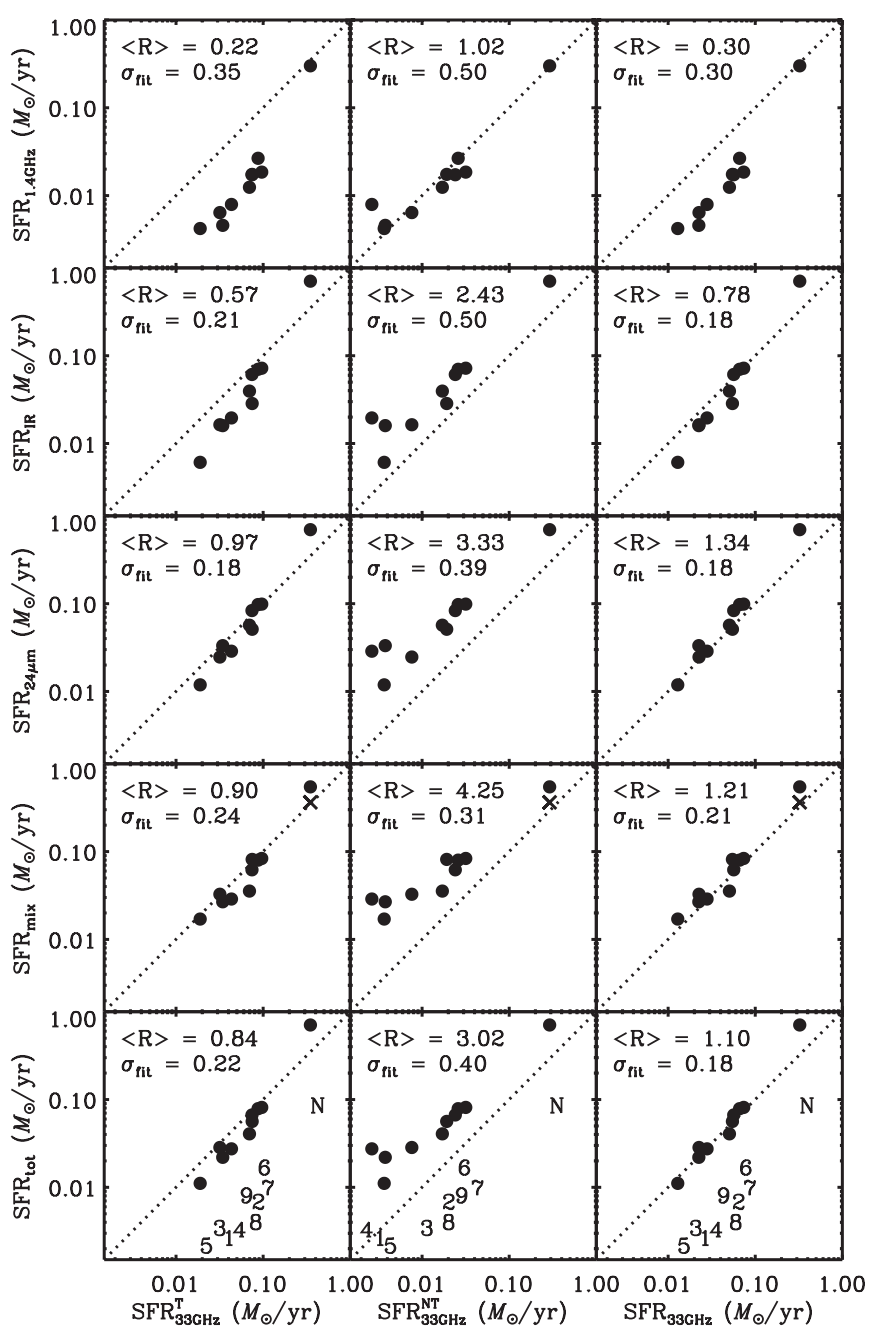

Figure 3. Comparison of the various extinction-independent SFR diagnostics described in Section 3 (i.e., $1.4 \mathrm{GHz}$ via the FIR-radio correlation, total IR, $24 \mu \mathrm{m}, \mathrm{H} \alpha+24 \mu \mathrm{m}-$ mix, and UV + IR - tot), plotted from left to right against the $33 \mathrm{GHz}$ free-free, non-thermal, and total radio SFRs, respectively. To help guide the eye, the one-to-one line is shown as a dotted line in each panel. The points are also identified per column in the bottom row of panels by the extranuclear region number and an " $N$ " for the nucleus. In the upper left corner of each panel, the median ratio of the ordinate to abscissa $(\langle R\rangle)$, along with the residual dispersion about an ordinary least-squares fit to each trend $\left(\sigma_{\text {fit }}\right)$, is given. In the plots comparing the $\mathrm{H} \alpha+24 \mu \mathrm{m}$ (mix) and $33 \mathrm{GHz}$ SFRs, we additionally plot the nucleus using the $\mathrm{H} \alpha+24 \mu \mathrm{m}$ calibration for entire galaxies (Kennicutt et al. 2009) as a cross.

mentioned, we only include SFR diagnostics whose reliability is considered to be fairly insensitive to extinction. SFRs estimated by free-free emission at $33 \mathrm{GHz}$ are assumed to be the most accurate and thus act as the reference SFR for the comparison. This assumption is made as the thermal fraction at $33 \mathrm{GHz}$ is found to be $\approx 87 \%$, on average. The various SFR diagnostics are plotted in Figure 3 from left to right against the $33 \mathrm{GHz}$ free-free, non-thermal, and total radio SFRs, respectively. The median ratio of the ordinate to abscissa $(\langle R\rangle)$, along with the residual dispersion after subtracting out an ordinary leastsquares fit to the data $\left(\sigma_{\text {fit }}\right)$, is given in the top left corners of each panel.

\subsection{Non-thermal Radio Emission: $1.4 \mathrm{GHz}$ Emission and the FIR-Radio Correlation}

In the top three panels of Figure 3, SFRs derived using the observed 1.4 GHz flux densities (along with the FIR-radio correlation) from each star-forming region are plotted against the three $33 \mathrm{GHz}$ derived estimates. Except for the starbursting nucleus, the $1.4 \mathrm{GHz}$ SFRs appear to significantly underestimate (i.e., by a factor of $\sim 5$ ) the actual SFR as measured by the free-free emission at $33 \mathrm{GHz}$. As stated at the end of Section 3.2, this discrepant behavior is expected given that the FIR-radio correlation applies to galaxies as a whole. The agreement for the nucleus likely arises from the fact that the $33 \mathrm{GHz}$ thermal fraction (i.e., $\sim 62 \%$ ) is significantly smaller than that of the extranuclear star-forming regions, being much more similar to what is found for globally integrated measurements of galaxies (e.g., Condon 1992). Differences between the radio and optical properties (e.g., $1.4 \mathrm{GHz}$ to $\mathrm{H} \alpha$ ratios) of galaxy nuclei and $\mathrm{H}$ II regions have been noted in the past by a number of authors (e.g., Israel \& Kennicutt 1980; Heckman et al. 1983; van der Hulst et al. 1988). These differences appear to be due to a larger nonthermal fraction in the nucleus relative to what is estimated for the extranuclear regions as previously suggested by Kennicutt et al. (1989), rather than simply higher optical extinctions.

Comparing the $1.4 \mathrm{GHz}$ SFRs with the $33 \mathrm{GHz}$ SFRs calculated using the total $33 \mathrm{GHz}$ flux densities (and Equation (15)), we again find that the $1.4 \mathrm{GHz}$ SFR calibrations are underestimating the SFR as measured by the total $33 \mathrm{GHz}$ flux density among the extranuclear regions. This is similar to the discrepancy between the $1.4 \mathrm{GHz}$ and $33 \mathrm{GHz}$ free-free SFRs, and most likely due to the fact that the thermal fractions are large at $33 \mathrm{GHz}$ among these star-forming regions. On the other hand, the $1.4 \mathrm{GHz}$ SFRs are nearly equal to those measured using the non-thermal $33 \mathrm{GHz}$ flux densities among both the extranuclear regions and the nucleus. This is not too surprising given that the $1.4 \mathrm{GHz}$ flux density is dominated by non-thermal emission and suggests that our thermal decomposition is in fact fairly robust. The only region where there is a significant difference is extranuclear region 4, however, the thermal/non-thermal decomposition here is complicated by the presence of an anomalous emission component.

\subsection{Total Infrared}

The second row of panels in Figure 3 compares the IR-based SFRs to those at $33 \mathrm{GHz}$. We find that the IR-based SFRs typically underestimate the SFRs derived from the free-free emission among the extranuclear regions by an average factor of $\sim 2$. There may also be a slight trend of increased discrepancy between the two SFR indicators with decreasing infrared surface brightness. This discrepancy among the extranuclear regions likely arises from the subtraction of a local background together with the application of an SFR calibration that assumes all UV photons are absorbed by dust at the source, which may not be true, since a significant fraction of non-ionizing photons can escape the star-forming complexes, powering the diffuse interstellar dust emission. That is, the lower surface brightness extranuclear star-forming regions may become increasingly porous to UV photons. Thus, by subtracting a local background, which makes up a significant fraction of the cool/cold dust emission, the IR-based SFR calibration yields values that are significantly lower than the free-free emission estimates. Similar results are seen when comparing the IR and radio SFRs using the total $33 \mathrm{GHz}$ flux densities.

On the other hand, the nucleus is a factor of $\sim 2$ times larger than the estimate using the free-free or total $33 \mathrm{GHz}$ emission. One reason for the large discrepancy in the nuclear SFR could arise from a significant amount of dust being heated by stars that are not contributing significantly to the ionization of 
$\mathrm{H}$ atoms. Alternatively, this difference may arise from the capture of ionizing photons by dust, which would decrease the free-free emission at $33 \mathrm{GHz}$ and increase the IR emission. A detailed discussion about such scenarios is given in Section 5.1.

The IR-based SFRs are generally larger (i.e., by a factor of more than $\sim 2$, on average) than the non-thermal $33 \mathrm{GHz}$ SFRs for all star-forming regions. This is consistent with the above $1.4 \mathrm{GHz}$ SFR comparison among the extranuclear regions which suggest that the SFR calibration based on the non-thermal radio emission typically underestimates the true SFR in such regions. This finding is also in agreement with studies of the resolved FIR-radio correlation within galaxies that have reported elevated FIR/radio ratios relative to the canonical correlation value for individual star-forming regions (e.g., Murphy et al. 2006a, 2008; Hughes et al. 2006). The physical explanation for this observation is that CR electrons propagate significantly further than dust-heating photons, thus the non-thermal radio continuum emission associated with individual star-forming regions is much more spread out and diffuse than the dust emission-an effect which is not taken into account by the SFR recipe (see Section 5.2).

\subsection{Warm Dust: 24 um Emission}

Focusing on only the warm dust emission, we compare $24 \mu \mathrm{m}$-derived SFRs against the $33 \mathrm{GHz}$ suite of SFRs in the third row of panels in Figure 3. Unlike the total IR-based SFRs, there is much better agreement between the $24 \mu \mathrm{m}$-derived and free-free emission SFRs among all extranuclear star-forming regions. There is also relatively good agreement among the $24 \mu \mathrm{m}$-derived and total $33 \mathrm{GHz}$ SFRs among all extranuclear star-forming regions, while the $24 \mu \mathrm{m}$-derived SFR for the nucleus is a factor of $\sim 2$ larger than each of the $33 \mathrm{GHz}$ SFR estimates. Similar to the discrepancy between the IR-based and $33 \mathrm{GHz}$ free-free SFRs for the nucleus, we may expect the $24 \mu \mathrm{m}$ derived and $33 \mathrm{GHz}$ free-free SFRs to also disagree because of either the capturing of ionizing photons by dust or enhanced heating of very small grains (see Section 5.1). Such grains are stochastically heated and only depend on the strength of the radiation field energy density. Warm dust may also arise from active galactic nuclei (AGNs) activity; however, there is currently no strong evidence for AGN activity in the nucleus of NGC 6946 (e.g., Tsai et al. 2006), and, like other comparisons with the non-thermal SFRs, the $24 \mu \mathrm{m}$ derived SFRs are a factor of $\sim 3$ larger than the non-thermal $33 \mathrm{GHz}$ SFRs, on average.

\subsection{Extinction-corrected $H \alpha: H \alpha+24 \mu m$}

The hybrid $\mathrm{H} \alpha+24 \mu \mathrm{m}$ SFR diagnostic has been found to be quite reliable relative to SFRs measured using extinctioncorrected $\mathrm{Pa} \alpha$ recombination line observations (e.g., Hubble Space Telescope NICMOS narrowband imaging; Calzetti et al. 2007). In the fourth row of panels, we plot this SFR diagnostic against each of the $33 \mathrm{GHz}$ SFRs, keeping in mind that we believe that the free-free emission likely gives the most reliable SFR. We find a similar behavior here as with the SFRs derived using warm dust emission. The $\mathrm{H} \alpha+24 \mu \mathrm{m}$ SFRs agree quite well with the $33 \mathrm{GHz}$ free-free SFRs for the extranuclear starforming regions, however, it is slightly larger than (i.e., 60\% above) the $33 \mathrm{GHz}$ thermal estimate in the nucleus. We find the same behavior in the comparison between the $\mathrm{H} \alpha+24 \mu \mathrm{m}$ and total $33 \mathrm{GHz}$ SFRs. The non-thermal $33 \mathrm{GHz}$ SFRs again underestimate the SFRs among the extranuclear star-forming regions relative to the $\mathrm{H} \alpha+24 \mu \mathrm{m}$ SFRs by an average factor of $\sim 4$.

\subsection{Bolometric Estimates: $U V+I R$}

Finally, we compare our last extinction-insensitive SFR diagnostic (i.e., UV + IR SFRs) with the $33 \mathrm{GHz}$ SFRs in the bottom panels of Figure 3. In general, we find that the UV + IR SFRs are in good agreement with the $33 \mathrm{GHz}$ free-free SFRs among the extranuclear star-forming regions. The case is marginally improved when comparing the UV + IR SFRs to the total $33 \mathrm{GHz}$ SFRs. Not surprisingly, we again find that the $33 \mathrm{GHz}$ non-thermal SFRs are quite discrepant, begin a factor of nearly $\sim 3$ lower than the UV + IR SFRs, and in all three comparisons we find that the nucleus UV + IR SFR is a factor of $\gtrsim 2$ larger than the $33 \mathrm{GHz}$ SFRs.

\section{DISCUSSION}

In the previous section, we quantitatively compared a number of SFR diagnostics whose reliability is considered to be largely insensitive to interstellar extinction for 10 star-forming regions within the nearby galaxy, NGC 6946. Using these results, we attempt to construct a self-consistent picture to explain the observed discrepancies between the SFR estimates for the nuclear and extranuclear regions by using free-free, dust, and non-thermal emission processes as diagnostics. Additionally, we discuss how combinations of these diagnostics can be used to infer other physical characteristics from star-forming regions and star-forming galaxies as a whole.

\subsection{Anomalous Behavior of the Nuclear Starburst}

One of the most striking differences in the present comparison of these star-forming regions is the behavior between the nucleus and the extranuclear star-forming regions. While the $1.4 \mathrm{GHz}$ radio continuum emission and the FIR-radio correlation yield an SFR that agrees well with the $33 \mathrm{GHz}$ free-free SFR for the nucleus, we find that all other diagnostics that rely on some sort of dust emission yield SFRs that are significantly larger than that when using the $33 \mathrm{GHz}$ free-free emission.

As previously stated, one way to explain the dust-based SFRs being larger than the $33 \mathrm{GHz}$ free-free SFR estimates in the nucleus is through dust capture of ionizing photons, which will in turn increase the dust emission while decreasing the $\mathrm{H} \alpha$ and free-free emission per unit star formation. The extinction in NGC 6946's nuclear starburst has been measured to be $A_{V} \sim 4.3 \mathrm{mag}\left(8^{\prime \prime} .5-280 \mathrm{pc}\right.$; Engelbracht et al. 1996) and $A_{V} \sim 4.6 \mathrm{mag}$ (2".7-90 pc; Quillen \& Yukita 2001) using Balmer decrements. Similar values have been estimated using the $9.7 \mu \mathrm{m}$ silicate absorption feature in the central kpc ( $A_{V} \sim 5.0$ mag; Smith et al. 2007), while much larger estimates have been quoted for the central $\sim 60$ pc using total gas masses $\left(A_{V} \sim 100 \mathrm{mag}\right.$; Schinnerer et al. 2006), indicating a highly obscured, compact component. Although the extinction is high toward the central starburst, the effect of dust capturing ionizing photons may not significantly affect our measurements over the entire $\sim 0.8 \mathrm{kpc}$ area covered by the GBT beam unless the starburst is highly concentrated within the central $\sim 60 \mathrm{pc}$.

Using our $24 \mu \mathrm{m}$ image at its native resolution, we find that $\sim 25 \%$ of this warm dust emission arises within the central 200 pc. Assuming that $50 \%-100 \%$ of this emission from the central starburst region (i.e., $12.5 \%-25 \%$ of the total $24 \mu \mathrm{m}$ emission) is powered by ionizing photons, which are thus not participating in the production of free-free emission, this does not account for the factor of $\sim 2$ discrepancy between the SFR estimates as the $24 \mu \mathrm{m}$ SFR would still be larger than the $33 \mathrm{GHz}$ free-free SFR by $\sim 55 \%-20 \%$, respectively. This estimate relies 
on the relation between the $24 \mu \mathrm{m}$ and free-free emission given by Equation (2) in Murphy et al. (2006b). It is also worth pointing out that the non-thermal (e.g., $1.4 \mathrm{GHz}$ ) emission should not be affected by this competition for ionizing photons and that these SFRs (i.e., the $1.4 \mathrm{GHz}$ and $33 \mathrm{GHz}$ non-thermal) agree with the $33 \mathrm{GHz}$ free-free SFR. Thus, it seems that dust absorption of ionizing photons by itself cannot explain this discrepancy.

Alternatively, it may be more likely that these relatively larger dust-based SFRs are the result of extra dust heating in the nucleus not associated with current star formation activity due to its large stellar density. In this case, the dust-based SFR will overestimate the true SFR for the nucleus of NGC 6946. Such a scenario seems plausible given the large stellar densities associated with the pseudobulge of NGC 6946 (Kormendy et al. 2010) which completely fills our beam; the radius of the bulge is $\approx 15^{\prime \prime}$ (Regan \& Vogel 1995) and contains a stellar minibar (Elmegreen et al. 1998). Furthermore, excess heating of dust (small and large grains) may arise from an accumulation of non-ionizing stars in the nucleus as the result of an extended duration of star formation activity associated with the starburst. Spectroscopic studies of near-infrared recombination lines have found that star formation episodes in nuclei differ significantly from those in extranuclear $\mathrm{H}$ II regions. Unlike extranuclear star-forming regions, galaxy nuclei typically show a spatial coincidence between the continuum and line emission suggesting that nuclear star formation activity is typically ongoing, where as the star-forming episodes in extranuclear regions are much younger and more transient in nature (Dale et al. 2004).

\subsubsection{Galaxy Nuclei Behaving More Like Entire Galaxies}

Assuming that ionizing photons are not being captured by dust, it is interesting to point out that if we instead extinction correct the $\mathrm{H} \alpha$ line emission using the relation given by Kennicutt et al. (2009) for entire galaxies, which scales the $24 \mu \mathrm{m}$ emission by a lower value $(0.020$ versus 0.031 for $\mathrm{H}$ II regions), we find good agreement between the $\mathrm{H} \alpha+24 \mu \mathrm{m}$ and $33 \mathrm{GHz}$ free-free SFRs (crosses plotted in Figure 3). Thus, it appears that the nucleus may behave more like an entire galaxy with respect to the non-thermal radio and $\mathrm{H} \alpha+24 \mu \mathrm{m}$ SFR calibrations. Assuming then, that our UV + IR SFR calibration is only appropriate for extranuclear star-forming complexes, we can set our $33 \mathrm{GHz}$ free-free SFR equal to Equation (8) to derive a new scaling to correct down the UV + IR SFR for the nucleus such that

$$
\left(\frac{\mathrm{SFR}_{\mathrm{tot}}^{\mathrm{nuc}}}{M_{\odot} \mathrm{yr}^{-1}}\right)=4.42 \times 10^{-44}\left(\frac{L_{\mathrm{FUV}}+0.43 L_{\mathrm{IR}}}{\mathrm{erg} \mathrm{s}^{-1}}\right)
$$

The coefficient scaling the IR emission is actually more in line with a detailed study of UV and IR emission from global measurements of normal star-forming galaxies, which report a value of $\sim 0.46$ (Hao et al. 2011).

Admittedly, the fact that the FIR-radio correlation appears to hold for the nucleus, and the non-thermal radio (i.e., $1.4 \mathrm{GHz}$ and $33 \mathrm{GHz}$ non-thermal) SFRs agree with the $33 \mathrm{GHz}$ free-free SFR here, could be fortuitous. Ultimately, it seems impossible to completely disentangle these two scenarios as there is no alternative way to accurately measure the ionizing photon rate. Thus, for such highly obscured regions, it appears difficult to accurately measure SFRs to better than a factor of $\sim 2$.

\subsection{Non-thermal Emission, SFRs, and CRs}

The fact that the prescriptions for converting the non-thermal emission into an SFR are doing a reasonable job for the nucleus relative to the extranuclear star-forming regions may be the result of the time dependence on CR electron diffusion. Work on the propagation of $\mathrm{CR}$ electrons in external galaxies has found a trend of decreasing CR electron propagation length with increasing radiation field energy density, as measured by the infrared surface brightness (Murphy et al. 2006b, 2008). The radiation field energy density in the nucleus, which appears to be in rough equipartition with the magnetic field energy density, is a factor of nearly $\sim 25$ times larger than that of the extranuclear star-forming regions, on average. This is consistent with a scenario of underestimated $1.4 \mathrm{GHz}$ SFRs in the extranuclear star-forming regions relative to the nucleus. We can see if this scenario is valid by comparing the cooling efficiencies and diffusion timescales for the nucleus and extranuclear starforming regions.

Let us assume that the propagation of CR electrons is described by a random walk with an energy-dependent diffusion coefficient $D_{E}$ (e.g., Ginzburg et al. 1980), where $D_{E}=5 \times$ $10^{28} \mathrm{~cm}^{2} \mathrm{~s}^{-1}$ for $E<1 \mathrm{GeV}$ and $5 \times 10^{28}(E / \mathrm{GeV})^{1 / 2} \mathrm{~cm}^{2} \mathrm{~s}^{-1}$ for $E \geqslant 1 \mathrm{GeV}$ (e.g., Jones et al. 2001; Moskalenko et al. 2002; Maurin et al. 2002). Then, the time it takes to diffuse a distance $l_{\text {diff }}$ is given by $\tau_{\text {diff }}=l_{\text {diff }}^{2} / D_{E}$. For $1.4 \mathrm{GHz}$ emitting $\mathrm{CR}$ electrons to travel a distance of $\approx 0.8 \mathrm{kpc}$ (i.e., to cross the GBT beam at $33 \mathrm{GHz}$ ), it will take $\sim 3.0$ and 2.4 Myr in the nucleus and extranuclear regions, on average, respectively. The slight difference in $\tau_{\text {diff }}$ arises from differences in the typical energy of $1.4 \mathrm{GHz}$ emitting electrons in the nucleus and extranuclear regions due to differences in the estimated magnetic field strengths. Accordingly, CR electrons in extranuclear star-forming regions will rapidly propagate away from their acceleration sites on timescales that are much longer than (i.e., a factor of $\sim 5$ ) estimates of their typical cooling times ( $\sim 12 \mathrm{Myr})$. On the other hand, the estimated cooling time for $1.4 \mathrm{GHz}$ emitting $\mathrm{CR}$ electrons in the nucleus is $\sim 2.0 \mathrm{Myr}$, which is $\sim 1.0$ Myr shorter than the estimated escape time.

Thus, the agreement between the non-thermal and $33 \mathrm{GHz}$ free-free SFRs for the nucleus likely arises from being in the calorimeter limit of Völk (1989) such that the continuously injected CR electrons, associated with the extended duration of star formation in the nucleus as discussed in Section 5.1, lose the bulk of their energy before propagating outside of the region covered by our beam. It is also worth noting that the large gas densities in the nucleus will lead to an increase in hadronic interactions between CR nuclei and the interstellar gas, netting more synchrotron radiation per unit star formation due to an increase in pionic secondaries $\left(e^{ \pm}\right)$. On the other hand, the transient nature of the star formation episodes in the young extranuclear star-forming complexes provides enough time for $\mathrm{CR}$ electrons to propagate significantly further (i.e., on the order of a $\sim \mathrm{kpc}$ ) than dust-heating and ionizing photons, resulting in an underestimate of the true SFR.

\subsubsection{Revised Non-thermal SFR Recipes for Individual Star-forming Regions}

It may be possible to correct the non-thermal SFR recipes by including a term that takes into account the time dependence of CR propagation. For example, through a detailed comparison between the non-thermal radio and infrared morphologies of nearby disk galaxies, it has been shown that $U_{\text {rad }}$ can be used 

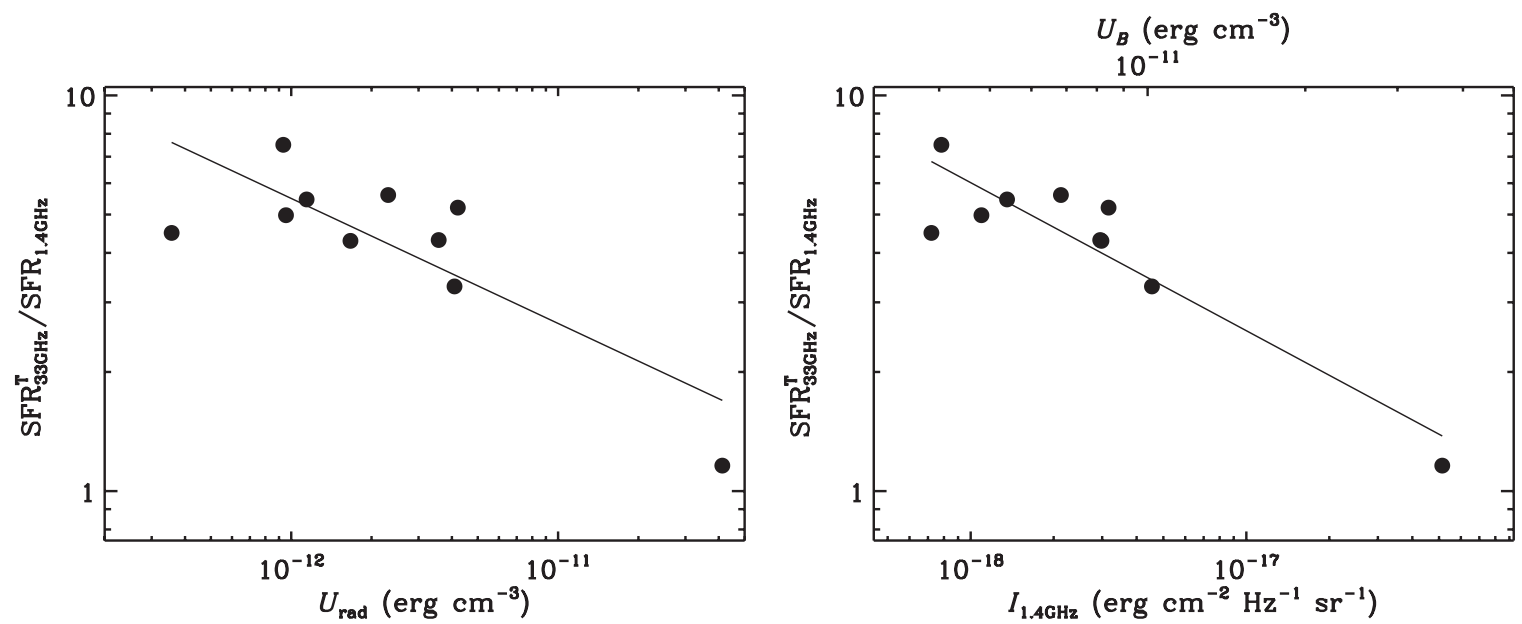

Figure 4. Ratio of the $33 \mathrm{GHz}$ free-free to $1.4 \mathrm{GHz}$ (non-thermal) SFRs plotted against $U_{\text {rad }}$ (left panel) and $1.4 \mathrm{GHz}$ surface brightness (right panel). Ordinary least-squares fits to the trends are shown.

as a proxy for the average distance traveled by the CR electrons (Murphy et al. 2006b, 2008). We therefore plot the ratio of the $33 \mathrm{GHz}$ free-free to $1.4 \mathrm{GHz}$ (non-thermal) SFRs against $U_{\mathrm{rad}}$ (left panel of Figure 4). We apply an ordinary least-squares fit to derive a rough correction for the two SFR recipes that rely on the non-thermal radio emission (i.e., Equations (14) and (17)). Accordingly, we can rewrite Equation (14) such that

$$
\begin{aligned}
\left(\frac{\mathrm{SFR}_{v}^{\mathrm{NT}-\text { corr }}}{M_{\odot} \mathrm{yr}^{-1}}\right) \sim & 5.89_{-5.5}^{+85} \times 10^{-32}\left(\frac{v}{\mathrm{GHz}}\right)^{\alpha^{\mathrm{NT}}}\left(\frac{L_{v}^{\mathrm{NT}}}{\operatorname{erg~s}^{-1} \mathrm{~Hz}^{-1}}\right) \\
& \times\left(\frac{U_{\mathrm{rad}}}{\mathrm{erg} \mathrm{cm}^{-3}}\right)^{-0.316 \pm 0.102},
\end{aligned}
$$

and Equation (17) such that

$$
\begin{aligned}
\left(\frac{\mathrm{SFR}_{1.4 \mathrm{GHz}}^{\mathrm{corr}}}{M_{\odot} \mathrm{yr}^{-1}}\right) \sim & 5.64_{-5.3}^{+81} \times 10^{-32}\left(\frac{L_{1.4 \mathrm{GHz}}}{\mathrm{erg} \mathrm{s}^{-1} \mathrm{~Hz}^{-1}}\right) \\
& \times\left(\frac{U_{\mathrm{rad}}}{\operatorname{erg~cm}{ }^{-3}}\right)^{-0.316 \pm 0.102}
\end{aligned}
$$

Uncertainties from the least-squares fits are given. Using these revised expressions results in $\left\langle\mathrm{SFR}_{33 \mathrm{GHz}}^{\mathrm{NT}-\text { corr }} / \mathrm{SFR}_{33 \mathrm{GHz}}^{\mathrm{T}}\right\rangle \sim$ $1.19 \pm 0.36$ and $\left\langle\mathrm{SFR}_{1.4 \mathrm{GHz}}^{\mathrm{corr}} / \mathrm{SFR}_{33 \mathrm{GHz}}^{\mathrm{T}}\right\rangle \sim 1.07 \pm 0.33$.

Since $U_{\text {rad }}$ should roughly scale with $U_{B}$ (e.g., given the tightness of FIR-radio correlation; Condon 1992), and $U_{B}$ scales as the non-thermal radio surface brightness, it is likely more convenient to try to derive a correction which relies on the nonthermal radio emission alone. We therefore plot the ratio of the $33 \mathrm{GHz}$ free-free to $1.4 \mathrm{GHz}$ (non-thermal) SFRs against the $1.4 \mathrm{GHz}$ surface brightness in the right panel of Figure 4 and perform a least-squares fit. This results in corrections to the SFR recipes relying on the non-thermal radio continuum where Equation (14) becomes

$$
\begin{aligned}
\left(\frac{\mathrm{SFR}_{v}^{\mathrm{NT}-\text { corr }}}{M_{\odot} \mathrm{yr}^{-1}}\right) \sim & 7.33_{-6.6}^{+64} \times 10^{-35}\left(\frac{v}{\mathrm{GHz}}\right)^{\alpha^{\mathrm{NT}}}\left(\frac{L_{v}^{\mathrm{NT}}}{\mathrm{WHz}^{-1}}\right) \\
& \times\left(\frac{I_{1.4 \mathrm{GHz}}}{\operatorname{erg~s}^{-1} \mathrm{~cm}^{-2} \mathrm{~Hz}^{-1} \mathrm{sr}^{-1}}\right)^{-0.374 \pm 0.057},
\end{aligned}
$$

and Equation (17) becomes

$$
\begin{aligned}
\left(\frac{\mathrm{SFR}_{1.4 \mathrm{GHz}}^{\mathrm{corr}}}{M_{\odot} \mathrm{yr}^{-1}}\right) \sim & 7.01_{-6.3}^{+61} \times 10^{-35}\left(\frac{L_{1.4 \mathrm{GHz}}}{\mathrm{erg} \mathrm{s}^{-1} \mathrm{~Hz}^{-1}}\right) \\
& \times\left(\frac{I_{1.4 \mathrm{GHz}}}{\mathrm{erg} \mathrm{s}^{-1} \mathrm{~cm}^{-2} \mathrm{~Hz}^{-1} \mathrm{sr}^{-1}}\right)^{-0.374 \pm 0.057} .
\end{aligned}
$$

These revised expressions result in $\left\langle\mathrm{SFR}_{33 \mathrm{GHz}}^{\mathrm{NT}-\text { corr }} / \mathrm{SFR}_{33 \mathrm{GHz}}^{\mathrm{T}}\right\rangle \sim$ $1.15 \pm 0.32$ and $\left\langle\mathrm{SFR}_{1.4 \mathrm{GHz}}^{\mathrm{corr}} / \mathrm{SFR}_{33 \mathrm{GHz}}^{\mathrm{T}}\right\rangle \sim 0.98 \pm 0.22$. Accordingly, these revised recipes may be more appropriate when estimating SFRs from individual star-forming regions within galaxies that have had local background emission removed.

\subsection{Physical Parameters}

If we assume that Equation (6) provides accurate (extinctioncorrected) $\mathrm{H} \alpha$ fluxes and that the thermal radio continuum has been precisely quantified, we can compare these two quantities to obtain a rough idea of the electron temperature in each starforming complex such that

$$
\begin{aligned}
\left(\frac{T_{\mathrm{e}}}{10^{4} \mathrm{~K}}\right) \sim & 3.68 \times 10^{23}\left[\left(\frac{S_{v}^{\mathrm{T}}}{\mathrm{erg} \mathrm{s}^{-1} \mathrm{~cm}^{2} \mathrm{~Hz}^{-1}}\right)\right. \\
& \left.\times\left(\frac{f_{\mathrm{H} \alpha}^{\mathrm{corr}}}{\mathrm{erg} \mathrm{s}^{-1} \mathrm{~cm}^{-2}}\right)^{-1}\left(\frac{v}{\mathrm{GHz}}\right)^{0.1}\right]^{1.695}
\end{aligned}
$$

(Caplan \& Deharveng 1986; Condon 1992). This relation assumes an ionized $\mathrm{He}$ to $\mathrm{H}$ density ratio of $N\left(\mathrm{He}^{+}\right) / N\left(\mathrm{H}^{+}\right) \sim$ 0.08 . The approximation for the temperature dependence of recombination line strengths is good to $\sim 1 \%$ over electron densities and temperatures ranging between $10^{2} \mathrm{~cm}^{-3} \lesssim n_{\mathrm{e}} \lesssim$ $10^{4} \mathrm{~cm}^{-3}$ and $5000 \mathrm{~K} \lesssim T_{\mathrm{e}} \lesssim 10,000 \mathrm{~K}$, respectively (Hummer \& Storey 1987; Condon 1992). Under these assumptions, the scatter measured by plotting $\mathrm{SFR}_{\text {mix }}$ versus $\mathrm{SFR}_{33 \mathrm{GHz}}^{\mathrm{T}}$ in Figure 3 is dominated by variations in the electron temperatures from each region.

The derived electron temperatures (see Table 4) range between $4000 \mathrm{~K} \lesssim T_{\mathrm{e}} \lesssim 14,000 \mathrm{~K}$, with a median of $\sim 11,000 \mathrm{~K}$, for all star-forming regions except for extranuclear regions 4 and 8 where unrealistically high temperatures are found, being 17,000 and $28,000 \mathrm{~K}$, respectively. There are two possible reasons for such an outcome. One possibility is that the empirical 
$\mathrm{H} \alpha$ extinction correction could be underestimating the true $\mathrm{H} \alpha$ luminosity in these regions. This scenario seems plausible given that the extinction correction is built upon an empirical correlation which has scatter and different calibration coefficients for different luminosity ranges.

Alternatively, the thermal fraction from these regions may be overestimated. We note that the $33 \mathrm{GHz}$ flux density of extranuclear region 8 was boosted due to potential oversubtraction of background emission which may not have been adequate. For extranuclear region 4 , the situation may be more complex as the $33 \mathrm{GHz}$ flux density from this source has been found to be a factor of $\sim 2$ larger than what one would estimate by fitting the lower frequency (i.e., $\lesssim 10 \mathrm{GHz}$ ) radio data. This excess emission is thought to arise from rapidly spinning ultrasmall grains having a non-zero electric dipole moment. It is possible that the subtraction of this component was underestimated, however, the presence of such an excess indicates a high column of dust, which may suggest a possible underestimate in the $\mathrm{H} \alpha$ extinction correction. It is also worth noting that an overprediction of the ionizing flux in extranuclear region 4 by the $33 \mathrm{GHz}$ free-free emission (i.e., after subtracting out the excess emission) relative to the $\mathrm{H} \alpha+24 \mu \mathrm{m}, 24 \mu \mathrm{m}$, and IR observations (e.g., see Figure 4) is opposite to the expectation if the excess emission was the result of optically thick free-free emission arising from a compact $\mathrm{H}$ II region. Presently, it is not possible to distinguish between these two scenarios.

It is also worth pointing out the low derived electron temperature for the nucleus. Similar to the discussion in Section 5.1, if we extinction correct the $\mathrm{H} \alpha$ using the relation given by Kennicutt et al. (2009) for entire galaxies, the electron temperature for the nucleus appears more reasonable, being $\sim 8000 \mathrm{~K}$.

\subsection{Applicability to Global Measurements of Star-forming Galaxies}

While the current analysis has focused on individual starforming complexes on $\sim 0.8 \mathrm{kpc}$ scales after removing a local diffuse background, it is instructive to investigate the significance of this subtraction in light of integrated measurements of star-forming galaxies for which a local subtraction is not possible. In Figure 5, we plot the same SFR comparisons as shown in Figure 3, except that the IR and radio SED fitting was done using the photometry without subtracting out a local diffuse background component (see the Appendix for the associated fits and derived parameters). To briefly summarize the results from fitting the radio data in this manner, the observed radio spectral indices are steeper, being $\approx 0.5$ on average, compared to $\approx 0.2$ on average, when a local background was removed. The $33 \mathrm{GHz}$ emission is still clearly dominated by free-free emission as the thermal fraction at $33 \mathrm{GHz}$ is only slightly smaller when including the local background emission, being $79 \%$, on average.

The trends found using these SFR estimates are nearly identical to those shown in Figure 3. The SFRs estimated using the non-thermal emission are still significantly underestimated relative to the $33 \mathrm{GHz}$ free-free SFRs among the extranuclear regions, albeit by an average factor of $\sim 2$ instead of $\sim 4-5$. This reduction in discrepancy is certainly due to the fact that the bulk of the non-thermal radio emission is diffuse. It then follows that the corrections derived for the non-thermal SFR recipes in Section 5.2 will only be applicable to discrete star-forming regions for which a local background has been removed. Refitting these trends using the quantities derived before background subtraction leads to new coefficients for the

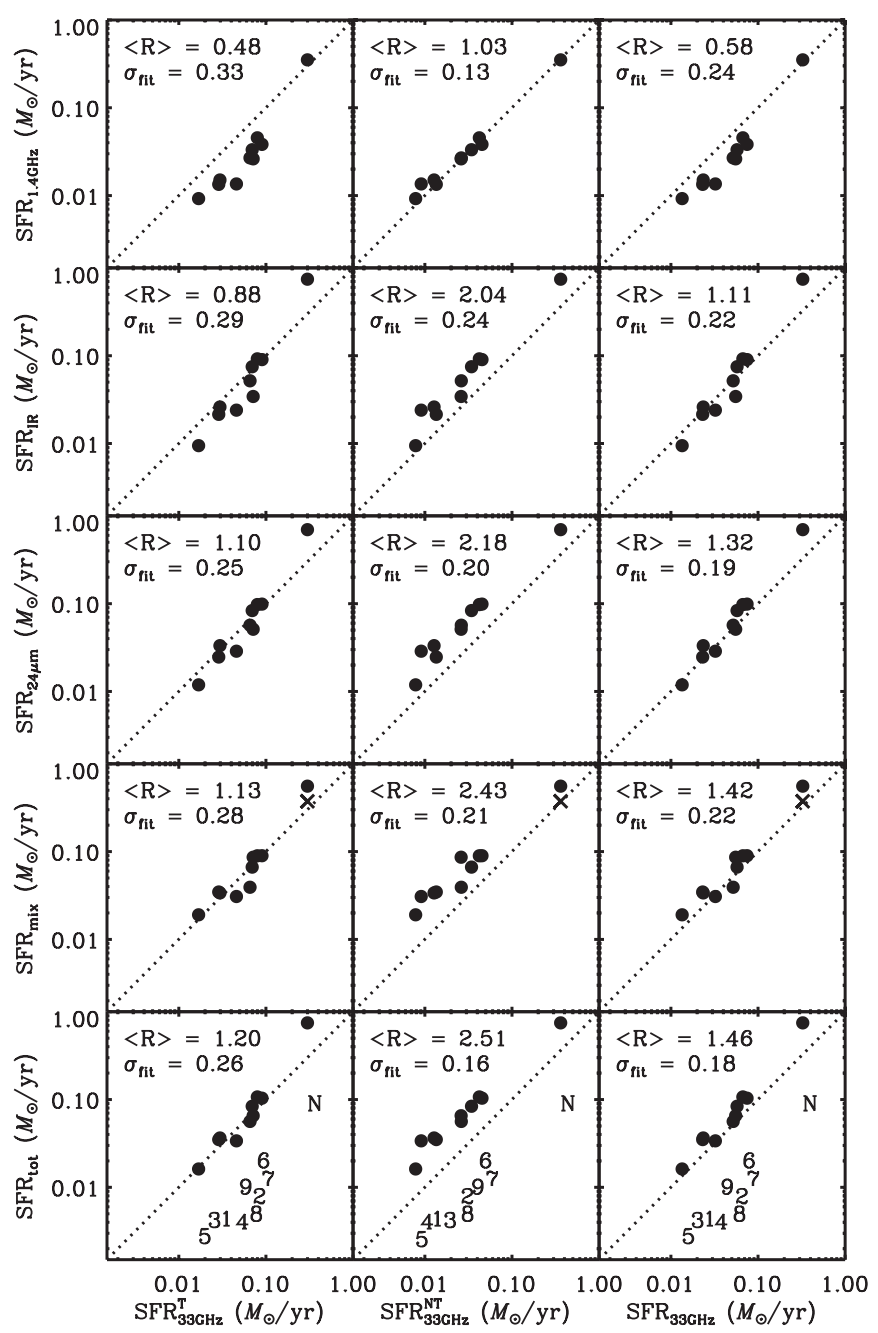

Figure 5. Same as Figure 3 except that SFRs have been calculated using the photometry without local background subtractions.

SFR recipes in Equations (19)-(22) of $5.35 \times 10^{-31}, 5.11 \times$ $10^{-31}, 3.31 \times 10^{-33}$, and $3.16 \times 10^{-33}$, respectively, while the exponents scaling $U_{\text {rad }}$ and $I_{1.4 \mathrm{GHz}}$ become -0.209 and -0.267 , respectively (see the Appendix for the rewritten expressions). Interestingly, we again find good agreement between the nonthermal SFRs and the $33 \mathrm{GHz}$ free-free SFR for the nucleus.

Among the SFRs which rely on some form of dust emission, we again find a reasonable agreement with the $33 \mathrm{GHz}$ free-free SFRs for the extranuclear star-forming regions. One notable difference is that the IR-based SFRs appear to be in much better agreement with the free-free emission SFRs by not having subtracted a local background. We also find that the nuclear SFRs are larger than the $33 \mathrm{GHz}$ free-free SFR for the nucleus by a factor of $\sim 2.5$, on average. So, while the sample presented here has targeted bright, active star-forming regions and is by no means representative of the global emission from a normal star-forming galaxy, the $\sim 0.8 \mathrm{kpc}$ scales investigated here do average over a number of discrete star formation sites which likely have a range in ages. Given this, and how well these diagnostics seem to agree even when including local diffuse emission, it appears that applying these diagnostics to entire systems which are vigorously forming stars is appropriate. In fact, it has recently been shown by Calzetti et al. (2010) that the $\mathrm{H} \alpha+24 \mu \mathrm{m}$ calibration used here, and derived for $\mathrm{H}$ II regions, works well for local IR-bright starbursts. Similarly, if we instead 


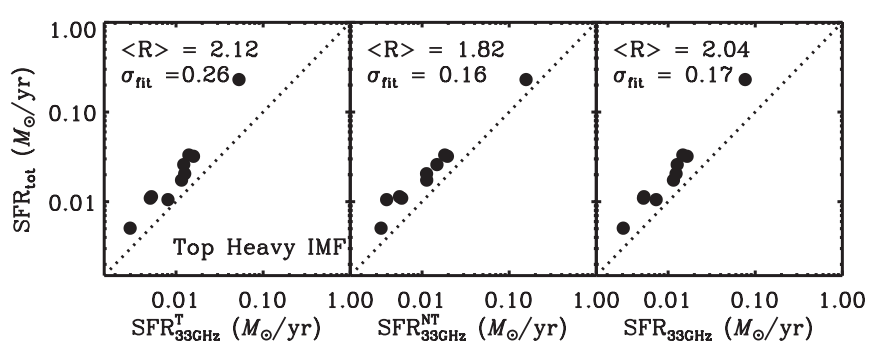

Figure 6. Same as the bottom three panels in Figure 5, except that a top-heavy Kroupa IMF was used, where the slope of the mass function is -1.5 for stellar masses ranging between 0.5 and $100 M_{\odot}$.

use the $\mathrm{H} \alpha+24 \mu \mathrm{m}$ calibration derived for entire galaxies (i.e., Kennicutt et al. 2009), which has been found to be appropriate for the $24 \mu \mathrm{m}$ luminosities of each region (Calzetti et al. 2010), when local backgrounds are not removed, these SFRs agree extremely well with the $33 \mathrm{GHz}$ free-free SFRs (i.e., median ratio near unity) over the entire luminosity range investigated. Of course, applying such diagnostics to entire galaxies must be done with care.

\subsection{A Test for IMF Variations in High-z Galaxies}

Recent work on low surface brightness dwarf galaxies has uncovered discrepancies between SFR diagnostics which depend on the ionizing photon rate (i.e., extinction-corrected $\mathrm{H} \alpha$ ) and the UV continuum (e.g., Meurer et al. 2009; Lee et al. 2009). One scenario to explain these discrepancies is a change in the upper end of the IMF of these systems since the ionizing photon rate is much more sensitive to changes in the high-mass end of the IMF than the UV continuum. Following the same logic, it may be possible to measure such discrepancies, and identify variations in the upper end of the IMF, in high- $z$ objects using future high-frequency ( $v \gtrsim 10 \mathrm{GHz}$ ) radio surveys, although it is worth noting that such variations can be mimicked by age effects, stochastic sampling of the IMF, and ionizing photon escape. The radio may be particularly powerful since, as we have shown, at higher frequencies the radio emission from galaxies becomes dominated by free-free emission. Surveys in the $\mathrm{X}$ band $(8-12 \mathrm{GHz})$ can probe the rest-frame $30 \mathrm{GHz}$ emission from star-forming galaxies at $z \sim 2$. In addition to measuring higher rest-frame frequencies with increasing redshift, the non-thermal component of a galaxy's radio continuum emission is expected to become increasingly suppressed with increasing redshift due to rapid cooling of CR electrons off of the cosmic microwave background (Murphy 2009). Hints of such an effect have been observed (e.g., flat radio spectral indices of lensed, high- $z$, galaxies; Ivison et al. 2010; Ikarashi et al. 2011). This additional suppression of the non-thermal emission from galaxies could improve the situation by leaving only the thermal component detectable.

In Figure 6, we present the same comparison of SFR diagnostics shown in the bottom three panels of Figure 5, except that top-heavy IMF calibrations have been used. We again run Starburst99 under the same assumptions as described in Section 3, except that the slope of the upper mass function has been flattened from -2.3 to -1.5 . We choose a top-heavy slope of -1.5 as this has been suggested as a way to explain the reionization of the intergalactic medium at $z \lesssim 11$ by star-forming galaxies (Chary 2008). In the associated output the ionizing flux increases by a factor of $\approx 6$ by moving to a top-heavy IMF, while the UV and IR emission only increases by a factor of $\approx 3$. One of course expects that SFRs that depend only on the ionizing flux should be similarly independent of the IMF. However, based on these differences in the calibrations, SFRs proportional to the ionizing flux should be a factor of $\sim 2$ times larger than those that depend on the UV and/or IR luminosities for a top-heavy IMF compared to a standard Kroupa IMF. In looking at Figure 6 this behavior is seen; the UV + IR SFRs yield an SFR which is nearly a factor of $\sim 2$ discrepant from the $33 \mathrm{GHz}$ thermal radio continuum estimate (i.e., a factor of $\sim 2$ larger since the IMF of NGC 6946 is more like a standard Kroupa IMF). Similarly, a systematic increase of $\sim 40 \%$ is found when comparing the IR+UV SFRs to the SFRs estimated from the total $33 \mathrm{GHz}$ radio continuum.

We note that in this last comparison, all three quantities used to estimate the SFRs are as observed; local backgrounds were not subtracted, no extinction correction was necessary, and it does not depend on an empirical calibration. Thus, by having high-quality UV, IR, and thermal radio continuum emission in hand, one should be able to quantify such systematic discrepancies between SFR diagnostics and search for sources with potentially top-heavy IMFs.

\section{CONCLUSIONS}

In the present analysis, we have investigated the applicability and reliability of a number of SFR diagnostics that are not hampered by extinction from dust. They include $1.4 \mathrm{GHz}$ (non-thermal) radio, IR, $24 \mu \mathrm{m}, \mathrm{H} \alpha+24 \mu \mathrm{m}$, and $\mathrm{UV}+\mathrm{IR}$ observations. This was done under the assumption that our free-free emission measurements in the $\mathrm{Ka}$ band (i.e., at $33 \mathrm{GHz}$ ) provide highly accurate estimates for the ionizing flux of massive star-forming regions. Although we only focus on 10 star-forming regions in a single galaxy (i.e., NGC 6946), the nuclear star-forming region is classified as a starburst and these regions span nearly two orders of magnitude in IR luminosity. Our main conclusions can be summarized as follows.

1. Excluding the total IR and non-thermal radio emission, there is very good agreement between the various diagnostics and the SFRs derived from the $33 \mathrm{GHz}$ free-free emission among the extranuclear star-forming regions, independent of local background subtractions. While the IR-based SFRs typically underestimate the SFRs derived from the free-free emission by an average factor of $\sim 2$ among the extranuclear star-forming regions, there is significantly better agreement between these two diagnostics when local backgrounds are not subtracted. These differences likely arise from the subtraction of a local background and the application of an SFR calibration that assumes all UV photons are absorbed by dust at the source.

2. Each of the diagnostics that rely on some sort of dust emission component yields SFRs for the nucleus that are larger than the $33 \mathrm{GHz}$ free-free emission SFR by an average factor of $\sim 2$. Given that the $1.4 \mathrm{GHz}$ SFR agrees with the $33 \mathrm{GHz}$ free-free emission SFR, and that only $\sim 25 \%$ of the $24 \mu \mathrm{m}$ emission within our beam arises from the central starburst, it seems most likely that these discrepancies are the result of excess IR heating arising from an accumulation of non-ionizing stars associated and extended duration of star formation in the nucleus rather than the capturing of ionizing photons by dust. However, conclusively distinguishing between these two scenarios is difficult.

3. Typical SFR recipes that rely on non-thermal radio continuum emission (e.g., 1.4 GHz SFRs based on the FIR-radio 
Table 5

Local Background Measurements

\begin{tabular}{|c|c|c|c|c|c|c|c|c|c|c|c|}
\hline Band & Nuc. & Enuc. 1 & Enuc. 2 & Enuc. 3 & Enuc. 4 & Enuc. 5 & Enuc. 6 & Enuc. 7 & Enuc. 8 & Enuc. 9 & $f_{\text {diff }}{ }^{\mathrm{a}}(\%)$ \\
\hline$S_{1.4 \mathrm{GHz}}(\mathrm{mJy})$ & 14.51 & 2.98 & 2.49 & 2.00 & 1.61 & 1.43 & 5.38 & 5.63 & 4.11 & 4.56 & 51 \\
\hline$S_{1.5 \mathrm{GHz}}(\mathrm{mJy})$ & 13.82 & 2.79 & 2.17 & 1.78 & 1.34 & 1.01 & 5.17 & 5.10 & 3.87 & 4.14 & 49 \\
\hline$S_{1.7 \mathrm{GHz}}(\mathrm{mJy})$ & 12.37 & 2.49 & 1.96 & 1.56 & 1.16 & 0.81 & 4.66 & 4.60 & 3.34 & 3.74 & 47 \\
\hline$S_{4.9 \mathrm{GHz}}(\mathrm{mJy})$ & 5.14 & 0.90 & 0.71 & 0.64 & 0.65 & 0.61 & 1.90 & 1.77 & 1.49 & 1.48 & 33 \\
\hline$S_{8.5 \mathrm{GHz}}(\mathrm{mJy})$ & 3.53 & 0.80 & 0.55 & 0.55 & 0.38 & 0.26 & 1.49 & 1.23 & 1.04 & 1.13 & 31 \\
\hline$f_{v}(850 \mu \mathrm{m})(\mathrm{mJy})$ & 42.0 & 1.2 & $\ldots$ & $\ldots$ & 0.0 & $\ldots$ & 4.3 & 2.7 & 0.0 & 3.6 & 5 \\
\hline$f_{\nu}(250 \mu \mathrm{m})(\mathrm{Jy})$ & 2.71 & 0.52 & 0.38 & 0.36 & 0.43 & 0.22 & 1.26 & 1.05 & 0.83 & 0.87 & 55 \\
\hline$f_{v}(160 \mu \mathrm{m})(\mathrm{Jy})$ & 6.48 & 1.03 & 0.74 & 0.54 & 0.63 & 0.40 & 2.81 & 2.31 & 1.66 & 1.83 & 38 \\
\hline$f_{v}(100 \mu \mathrm{m})(\mathrm{Jy})$ & 5.90 & 0.85 & 0.54 & 0.40 & 0.39 & 0.24 & 2.38 & 1.89 & 1.32 & 1.45 & 30 \\
\hline$f_{v}(70 \mu \mathrm{m})(\mathrm{Jy})$ & 3.12 & 0.37 & 0.22 & 0.17 & 0.13 & 0.06 & 1.23 & 0.89 & 0.57 & 0.66 & 21 \\
\hline$f_{v}(24 \mu \mathrm{m})(\mathrm{mJy})$ & 169.1 & 24.7 & 14.4 & 12.1 & 11.3 & 7.8 & 63.2 & 55.0 & 37.2 & 41.9 & 15 \\
\hline$f_{\nu}(8 \mu \mathrm{m})(\mathrm{mJy})$ & 170.6 & 32.4 & 18.3 & 17.2 & 13.4 & 13.2 & 77.2 & 68.3 & 50.8 & 54.8 & 28 \\
\hline$f_{\mathrm{H} \alpha}\left(10^{-12} \mathrm{erg} \mathrm{s}^{-1} \mathrm{~cm}^{-2}\right)$ & 0.34 & 0.24 & 0.16 & 0.07 & 0.07 & 0.07 & 0.32 & 0.21 & 0.13 & 0.15 & 16 \\
\hline$f_{v}(2271 \AA)(\mathrm{mJy})$ & 1.74 & 1.88 & 1.67 & 0.62 & 0.94 & 0.83 & 2.86 & 2.01 & 1.82 & 1.67 & 40 \\
\hline$f_{\text {diff }^{\mathrm{a}}(\%)}$ & 14 & 49 & 21 & 29 & 22 & 39 & 40 & 32 & 46 & 37 & \\
\hline
\end{tabular}

Note. ${ }^{\text {a }}$ The average fractional contribution of the background emission at each waveband and region (i.e., the fraction of diffuse emission) is given at the end of each row and column, respectively.

correlation) underestimate the true SFRs by an average factor of $\sim 4-5$, or an average factor of $\sim 2$ when local backgrounds are not removed, among the extranuclear starforming regions. This is in contrast with the nucleus, for which the non-thermal emission estimates agree rather well with the $33 \mathrm{GHz}$ free-free emission. We believe that the agreement between the non-thermal and $33 \mathrm{GHz}$ free-free SFRs arises from CR electrons decaying within the nuclear starburst region with negligible escape, mirroring its high optical depth to ionizing photons. On the other hand, the transient nature of star formation episodes in the young extranuclear star-forming complexes allows for CR electrons to diffuse significantly further than dust-heating photons, resulting in an underestimate in the SFR using the nonthermal emission. To properly measure the SFRs from individual star-forming regions using non-thermal emission requires a revised SFR recipe that attempts to incorporate the time dependence of CR propagation.

4. Using the total $33 \mathrm{GHz}$ flux densities (i.e., not corrected for the presence of non-thermal emission) yields SFRs that are in excellent agreement with those measured using only the $33 \mathrm{GHz}$ free-free emission and combination of $\mathrm{H} \alpha+24 \mu \mathrm{m}$ data among the extranuclear star-forming complexes. This result arises from the fact that free-free emission dominates the total flux density at $33 \mathrm{GHz}$ (i.e., $\approx 87 \%$, on average, among the star-forming regions investigated) and suggests that observations in the rest-frame $\mathrm{Ka}$ band may provide accurate SFRs in galaxies without the need of ancillary radio data to account for the non-thermal component.

5. Even without correcting our photometry for local diffuse background emission, which is prominent for observations that are most sensitive to non-thermal radio or cold dust emission, the $33 \mathrm{GHz}$ flux densities are still dominated by free-free emission with thermal fractions of $\approx 79 \%$, on average. Furthermore, comparisons between the various SFR diagnostics remain generally unaffected, suggesting that these recipes are applicable to entire galaxies which are dominated by ongoing star formation activity.

6. Using a combination of high-frequency ( $v \gtrsim 10 \mathrm{GHz}$ ) radio data along with rest-frame far-infrared and UV data, one can compare radio versus UV + IR SFR estimates which should become increasingly discrepant (i.e., a factor of $\sim 2$ ) as the IMF shifts toward the one that is top heavy. This test makes use of the fact that radio continuum emission becomes dominated by thermal (free-free) emission at higher frequencies and, in addition, that the non-thermal emission of high- $z$ galaxies should become increasingly suppressed due to increased IC losses of CR electrons off of the cosmic microwave background. Thus, deep surveys at frequencies $\gtrsim 10 \mathrm{GHz}$ (e.g., $\mathrm{X}$ band; 8-10 GHz) should begin to detect the free-free emission from galaxies at $z \gtrsim 2$ directly. Such tests to identify variations in the IMF of high$z$ systems should be possible using deep survey imaging from upcoming radio (EVLA, MeerKAT, and SKA/NAA), millimeter/IR (ALMA/CCAT), and optical/NIR (James Webb Space Telescope) facilities.

We thank the referee, T. Takeuchi, for useful suggestions that helped to improve the content and presentation of this paper. E.J.M. thanks the Observatories of the Carnegie Institution of Washington, where the final version of this paper was written. The National Radio Astronomy Observatory is a facility of the National Science Foundation operated under cooperative agreement by Associated Universities, Inc. We are grateful to the SINGS team for producing high-quality data sets used in this study. This work is based in part on observations made with the SST, which is operated by the Jet Propulsion Laboratory, California Institute of Technology under a contract with NASA. Herschel is an ESA space observatory with science instruments provided by European-led Principal Investigator consortia and with important participation from NASA.

\section{APPENDIX}

\section{ESTIMATES OF LOCAL BACKGROUNDS AND THEIR EFFECT IN THE SED FITTING}

In Table 5, we provide the values used for the local background subtraction at each wavelength for all star-forming regions. We also give the average fractional contribution of the background emission to the total emission per each wavelength 
Table 6

Derived Parameters Calculated without Local Backgrounds Subtracted

\begin{tabular}{lcccccc}
\hline \hline ID & $\begin{array}{c}f_{\mathrm{T}}^{33 \mathrm{GHz}} \\
(\%)\end{array}$ & $\alpha^{\text {obsa }}$ & $\alpha^{\mathrm{NTa}}$ & $\begin{array}{c}L_{\mathrm{IR}} \\
\left(10^{8} L_{\odot}\right)\end{array}$ & $\begin{array}{c}B_{\min }{ }^{\mathrm{b}} \\
(\mu \mathrm{G})\end{array}$ & $\begin{array}{c}T_{\mathrm{e}} \\
\left(10^{4} \mathrm{~K}\right)\end{array}$ \\
\hline Nucleus & $53 \pm 6$ & $0.62 \pm 0.04$ & $0.74 \pm 0.05$ & $50.10 \pm 2.43$ & $31.6 \pm 9.7$ & $0.31 \pm 0.07$ \\
Enuc. 1 & $80 \pm 5$ & $0.51 \pm 0.04$ & $0.81 \pm 0.06$ & $1.76 \pm 0.11$ & $13.8 \pm 4.0$ & $0.72 \pm 0.21$ \\
Enuc. 2 & $83 \pm 6$ & $0.43 \pm 0.03$ & $0.83 \pm 0.06$ & $2.31 \pm 0.14$ & $15.9 \pm 4.6$ & $0.65 \pm 0.24$ \\
Enuc. 3 & $79 \pm 7$ & $0.48 \pm 0.04$ & $0.81 \pm 0.06$ & $1.45 \pm 0.10$ & $13.4 \pm 3.9$ & $0.65 \pm 0.25$ \\
Enuc. 4 & $45^{\mathrm{c}} \pm 5$ & $0.36 \pm 0.02$ & $0.81 \pm 0.06$ & $1.62 \pm 0.10$ & $13.4 \pm 3.9$ & $1.75 \pm 0.61$ \\
Enuc. 5 & $78 \pm 8$ & $0.49 \pm 0.04$ & $0.80 \pm 0.06$ & $0.64 \pm 0.06$ & $12.1 \pm 3.5$ & $0.73 \pm 0.32$ \\
Enuc. 6 & $79 \pm 6$ & $0.56 \pm 0.04$ & $0.84 \pm 0.06$ & $6.21 \pm 0.30$ & $18.4 \pm 5.3$ & $0.73 \pm 0.19$ \\
Enuc. 7 & $79 \pm 6$ & $0.48 \pm 0.04$ & $0.84 \pm 0.06$ & $6.08 \pm 0.31$ & $17.6 \pm 5.0$ & $0.90 \pm 0.22$ \\
Enuc. 8 & $82 \pm 6$ & $0.46 \pm 0.03$ & $0.83 \pm 0.06$ & $3.49 \pm 0.18$ & $16.0 \pm 4.6$ & $2.14 \pm 0.48$ \\
Enuc. 9 & $79 \pm 6$ & $0.50 \pm 0.04$ & $0.84 \pm 0.06$ & $5.05 \pm 0.26$ & $17.0 \pm 4.9$ & $0.96 \pm 0.23$ \\
\hline
\end{tabular}

Notes.

${ }^{a}$ The observed and non-thermal radio spectral indices measured between 1.4 and $8.5 \mathrm{GHz}$.

${ }^{\mathrm{b}}$ Calculated using the total $1.4 \mathrm{GHz}$ flux densities and the revised minimum energy calculation of Beck \& Krause (2005).

c Calculated using the observed $33 \mathrm{GHz}$ flux density before subtracting out the $\approx 50 \%$ contribution thought to arise from anomalous dust emission (see Section 2.4).

and region at the end of each row and column, respectively. These values provide an estimate of the typical amount of diffuse emission contributing to each wavelength and region. Not surprisingly, the low-frequency (i.e., $1.4,1.5$, and $1.7 \mathrm{GHz}$ ) radio observations, which are dominated by non-thermal emission, are comprised of $\sim 50 \%$ of diffuse emission, on average. The fractional contribution of the background to the total measurements at $850 \mu \mathrm{m}$ is anomalously low, which is most likely due to the insensitivity of these data. Comparing the average diffuse emission fraction among each star-forming complex, we see that the nucleus has the lowest contribution, being a factor of $\sim 2.5$ smaller at $14 \%$, on average.

Additionally, we show the modeled SEDs (Figure 7) and give the associated derived parameters (Table 6) of each starforming region using the photometry before subtracting out a local diffuse component. The observed radio spectral indices are much steeper by not subtracting out a local diffuse component, being $\approx 0.5$, on average, compared to $\approx 0.2$, on average, when the backgrounds are removed. This finding is consistent with the low-frequency non-thermal emission being dominated by a diffuse component. Also note worthy is the fact that the thermal fraction at $33 \mathrm{GHz}$ is still large, being $\approx 79 \%$, on average.

As described in Section 5.4, the corrections derived for the non-thermal SFR recipes in Section 5.2, which attempt to take into account the time dependence of CR propagation, will only be applicable to discrete star-forming regions for which a local background has been removed. By refitting these trends using the photometry without subtracting local backgrounds leads to different revised SFR recipes such that Equations (19) and (20) become

$$
\begin{aligned}
\left(\frac{\mathrm{SFR}_{v}^{\mathrm{NT}-\text { corr }}}{M_{\odot} \mathrm{yr}^{-1}}\right) \sim & 5.35_{-4.9}^{+58} \times 10^{-31}\left(\frac{v}{\mathrm{GHz}}\right)^{\alpha^{\mathrm{NT}}}\left(\frac{L_{v}^{\mathrm{NT}}}{\mathrm{erg} \mathrm{s}^{-1} \mathrm{~Hz}^{-1}}\right) \\
& \times\left(\frac{U_{\mathrm{rad}}}{\mathrm{erg} \mathrm{cm}^{-3}}\right)^{-0.209 \pm 0.094} \quad(\mathrm{~A} 1)
\end{aligned}
$$

and

$$
\begin{aligned}
\left(\frac{\mathrm{SFR}_{1.4 \mathrm{GHz}}^{\mathrm{corr}}}{M_{\odot} \mathrm{yr}^{-1}}\right) \sim & 5.11_{-4.7}^{+55} \times 10^{-31}\left(\frac{L_{1.4 \mathrm{GHz}}}{\mathrm{erg} \mathrm{s}^{-1} \mathrm{~Hz}^{-1}}\right) \\
& \times\left(\frac{U_{\mathrm{rad}}}{\operatorname{erg~cm}{ }^{-3}}\right)^{-0.209 \pm 0.094},
\end{aligned}
$$

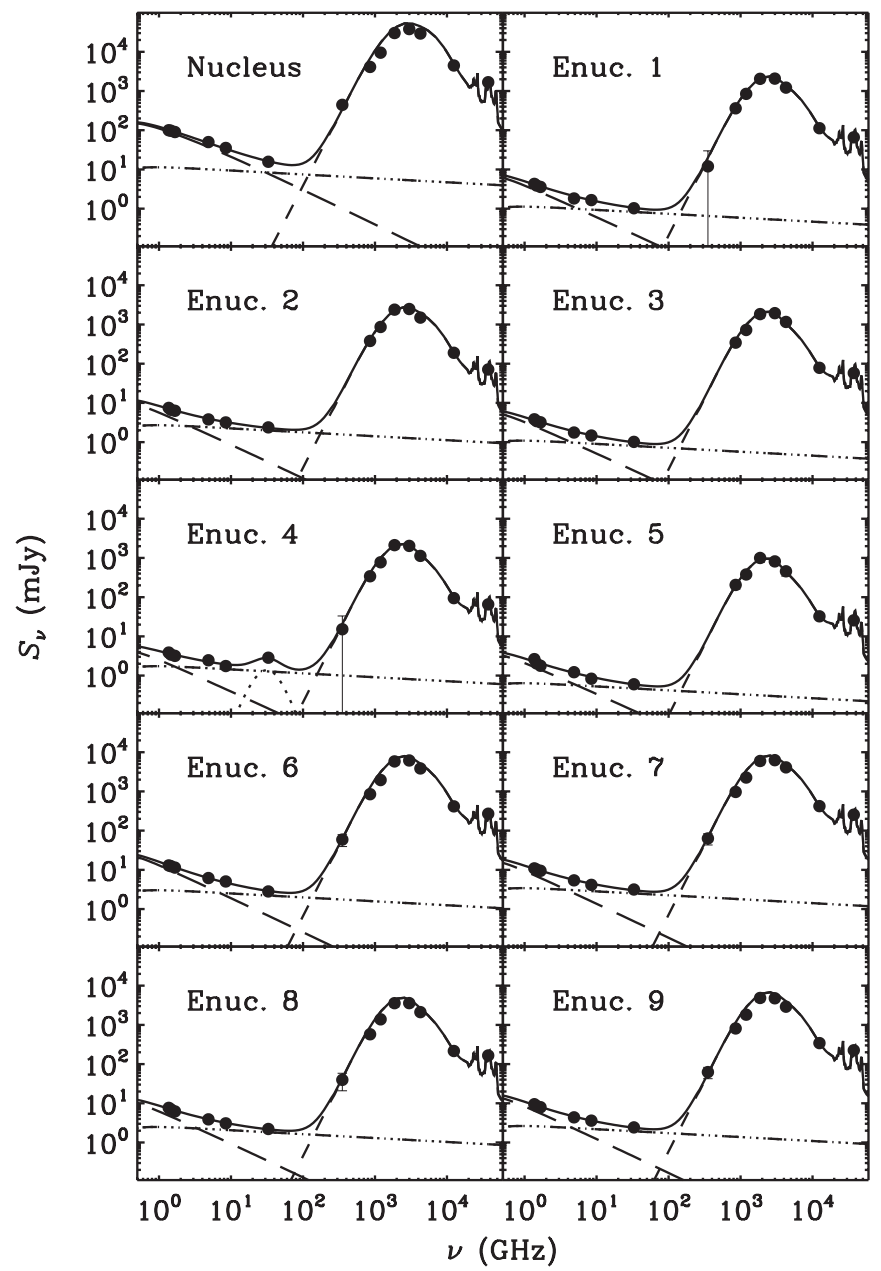

Figure 7. Same as Figure 2, except that the SEDs were fit using the photometry without subtracting out a local diffuse component.

respectively. Using these revised expressions results in $\left\langle\mathrm{SFR}_{33 \mathrm{GHz}}^{\mathrm{NT}-\text { corr }} / \mathrm{SFR}_{33 \mathrm{GHz}}^{\mathrm{T}}\right\rangle \sim 0.95 \pm 0.27$ and $\left\langle\mathrm{SFR}_{1.4 \mathrm{GHz}}^{\mathrm{corr}} /\right.$ $\left.\mathrm{SFR}_{33 \mathrm{GHz}}^{\mathrm{T}}\right\rangle \sim 1.03 \pm 0.29$. Similarly, by using the photometry before local background subtraction, Equations (21) and 
(22) become

$$
\begin{aligned}
\left(\frac{\mathrm{SFR}_{v}^{\mathrm{NT}-\mathrm{corr}}}{M_{\odot} \mathrm{yr}^{-1}}\right) \sim & 3.31_{-3.2}^{+75} \times 10^{-33}\left(\frac{v}{\mathrm{GHz}}\right)^{\alpha^{\mathrm{NT}}}\left(\frac{L_{v}^{\mathrm{NT}}}{\mathrm{WHz}^{-1}}\right) \\
& \times\left(\frac{I_{1.4 \mathrm{GHz}}}{\mathrm{erg} \mathrm{s}^{-1} \mathrm{~cm}^{-2} \mathrm{~Hz}^{-1} \mathrm{sr}^{-1}}\right)^{-0.267 \pm 0.080}
\end{aligned}
$$

and

$$
\begin{gathered}
\left(\frac{\mathrm{SFR}_{1.4 \mathrm{GHz}}^{\mathrm{corr}}}{M_{\odot} \mathrm{yr}^{-1}}\right) \sim 3.16_{-3.0}^{+72} \times 10^{-33}\left(\frac{L_{1.4 \mathrm{GHz}}}{\mathrm{erg} \mathrm{s}^{-1} \mathrm{~Hz}^{-1}}\right) \\
\quad \times\left(\frac{I_{1.4 \mathrm{GHz}}}{\mathrm{erg} \mathrm{s}^{-1} \mathrm{~cm}^{-2} \mathrm{~Hz}^{-1} \mathrm{sr}^{-1}}\right)^{-0.267 \pm 0.080}
\end{gathered}
$$

respectively. These revised expressions result in $\left\langle\mathrm{SFR}_{33 \mathrm{GHz}}^{\mathrm{NT}-\text { corr }}\right.$ / $\left.\mathrm{SFR}_{33 \mathrm{GHz}}^{\mathrm{T}}\right\rangle \sim 0.97 \pm 0.24$ and $\left\langle\mathrm{SFR}_{1.4 \mathrm{GHz}}^{\mathrm{corr}} / \mathrm{SFR}_{33 \mathrm{GHz}}^{\mathrm{T}}\right\rangle \sim 1.03 \pm$ 0.25 . Accordingly, these revised recipes may be more appropriate when estimating SFRs from individual star-forming regions within galaxies that have not had local backgrounds removed.

\section{REFERENCES}

Ali-Haïmoud, Y., Hirata, C. M., \& Dickinson, C. 2009, MNRAS, 395, 1055 Alonso-Herrero, A., Rieke, G. H., Rieke, M. J., et al. 2006, ApJ, 650, 835 Ball, R., Sargent, A. I., Scoville, N. Z., Lo, K. Y., \& Scott, S. L. 1985, ApJ, 298, L21

Beck, R. 2007, A\&A, 470, 539

Beck, R., \& Krause, M. 2005, Astron. Nachr., 326, 414

Bell, E. F. 2003, ApJ, 586, 794

Bendo, G. J., Wilson, C. D., Pohlen, M., et al. 2010, A\&A, 518, L65

Bouwens, R. J., Illingworth, G. D., Franx, M., et al. 2009, ApJ, 705, 936

Braun, R., Oosterloo, T. A., Morganti, R., Klein, U., \& Beck, R. 2007, A\&A, 461,455

Buat, V., Giovannoli, E., Burgarella, D., et al. 2010, MNRAS, 409, L1

Buat, V., Giovannoli, E., Takeuchi, T. T., et al. 2011, A\&A, 529, A22

Buat, V., Takeuchi, T. T., Iglesias-Páramo, J., et al. 2007, ApJS, 173, 404

Calzetti, D. 2001, PASP, 113, 1449

Calzetti, D., Kennicutt, R. C., Engelbracht, C. W., et al. 2007, ApJ, 666, 870

Calzetti, D., Kennicutt, Jr., R. C., Bianchi, L., et al. 2005, ApJ, 633, 871

Calzetti, D., Wu, S., Hong, S., et al. 2010, ApJ, 714, 1256

Caplan, J., \& Deharveng, L. 1986, A\&A, 155, 297

Chary, R. 2008, ApJ, 680, 32

Chary, R., \& Elbaz, D. 2001, ApJ, 556, 562

Condon, J. J. 1992, ARA\&A, 30, 575

Condon, J. J., \& Yin, Q. F. 1990, ApJ, 357, 97

Daddi, E., Dickinson, M., Morrison, G., et al. 2007, ApJ, 670, 156

Dale, D. A., \& Helou, G. 2002, ApJ, 576, 159

Dale, D. A., Roussel, H., Contursi, A., et al. 2004, ApJ, 601, 813

de Jong, T., Klein, U., Wielebinski, R., \& Wunderlich, E. 1985, A\&A, 147, L6

Draine, B. T. 2003, ARA\&A, 41, 241

Draine, B. T., \& Lazarian, A. 1998, ApJ, 508, 157

Dumas, G., Schinnerer, E., Tabatabaei, F. S., et al. 2011, AJ, 141, 41

Elbaz, D., Daddi, E., Le Borgne, D., et al. 2007, A\&A, 468, 33

Elmegreen, D. M., Chromey, F. R., \& Santos, M. 1998, AJ, 116, 1221

Engelbracht, C. W., Rieke, M. J., Rieke, G. H., \& Latter, W. B. 1996, ApJ, 467, 227

Ferguson, A. M. N., Wyse, R. F. G., Gallagher, J. S., \& Hunter, D. A. 1998, ApJ, 506, L19

Ginzburg, V. L., Khazan, I. M., \& Ptuskin, V. S. 1980, Ap\&SS, 68, 295

Gordon, K. D., Engelbracht, C. W., Rieke, G. H., et al. 2008, ApJ, 682, 336

Hao, C., et al. 2011, ApJ, submitted

Heckman, T. M., van Breugel, W., Miley, G. K., \& Butcher, H. R. 1983, AJ, 88, 1077

Helou, G. 1986, ApJ, 311, L33

Helou, G., Roussel, H., Appleton, P., et al. 2004, ApJS, 154, 253

Helou, G., Soifer, B. T., \& Rowan-Robinson, M. 1985, ApJ, 298, L7

Hirashita, H., Buat, V., \& Inoue, A. K. 2003, A\&A, 410, 83

Howell, J. H., Armus, L., Mazzarella, J. M., et al. 2010, ApJ, 715, 572

Hughes, A., Wong, T., Ekers, R., et al. 2006, MNRAS, 370, 363

Hummer, D. G., \& Storey, P. J. 1987, MNRAS, 224, 801
Iglesias-Páramo, J., Buat, V., Donas, J., Boselli, A., \& Milliard, B. 2004, A\&A, 419, 109

Iglesias-Páramo, J., Buat, V., Takeuchi, T. T., et al. 2006, ApJS, 164, 38

Ikarashi, S., Kohno, K., Aguirre, J. E., et al. 2011, MNRAS, 1143

Israel, F. P., \& Kennicutt, R. C. 1980, Astrophys. Lett., 21, 1

Ivison, R. J., Swinbank, A. M., Swinyard, B., et al. 2010, A\&A, 518, L35

Jones, F. C., Lukasiak, A., Ptuskin, V., \& Webber, W. 2001, ApJ, 547, 264

Karachentsev, I. D., Sharina, M. E., \& Huchtmeier, W. K. 2000, A\&A, 362, 544

Kennicutt, R. C., Hao, C., Calzetti, D., et al. 2009, ApJ, 703, 1672

Kennicutt, R. C., Jr. 1998, ARA\&A, 36, 189

Kennicutt, R. C., Jr., Keel, W. C., \& Blaha, C. A. 1989, AJ, 97, 1022

Kennicutt, R. C., Jr., Armus, L., Bendo, G., et al. 2003, PASP, 115, 928

Kennicutt, R. C., Jr., Calzetti, D., Walter, F., et al. 2007, ApJ, 671, 333

Klein, U., \& Graeve, R. 1986, A\&A, 161, 155

Klein, U., Wielebinski, R., \& Morsi, H. W. 1988, A\&A, 190, 41

Kobulnicky, H. A., \& Johnson, K. E. 1999, ApJ, 527, 154

Kormendy, J., Drory, N., Bender, R., \& Cornell, M. E. 2010, ApJ, 723, 54

Kroupa, P. 2001, MNRAS, 322, 231

Lee, J. C., Gil de Paz, A., Tremonti, C., et al. 2009, ApJ, 706, 599

Leitherer, C., Schaerer, D., Goldader, J. D., et al. 1999, ApJS, 123, 3

Li, Y., Calzetti, D., Kennicutt, R. C., et al. 2010, ApJ, 725, 677

Lonsdale Persson, C. J., \& Helou, G. 1987, ApJ, 314, 513

Mason, B. S., Weintraub, L., Sievers, J., et al. 2009, ApJ, 704, 1433

Maurin, D., Taillet, R., \& Donato, F. 2002, A\&A, 394, 1039

Meurer, G. R., Heckman, T. M., \& Calzetti, D. 1999, ApJ, 521, 64

Meurer, G. R., Wong, O. I., Kim, J. H., et al. 2009, ApJ, 695, 765

Mezger, P. G., \& Henderson, A. P. 1967, ApJ, 147, 471

Moskalenko, I. V., Strong, A. W., Ormes, J. F., \& Potgieter, M. S. 2002, ApJ, 565,280

Murphy, E. J. 2009, ApJ, 706, 482

Murphy, E. J., Chary, R.-R., Dickinson, M., et al. 2011, ApJ, 732, 126

Murphy, E. J., Helou, G., Kenney, J. D. P., Armus, L., \& Braun, R. 2008, ApJ, 678,828

Murphy, E. J., Braun, R., Helou, G., et al. 2006a, ApJ, 638, 157

Murphy, E. J., Helou, G., Braun, R., et al. 2006b, ApJ, 651, L111

Murphy, E. J., Helou, G., Condon, J. J., et al. 2010, ApJ, 709, L108

Ott, S. 2010, in ASP Conf. Ser. 434, Astronomical Data Analysis Software and Systems XIX, ed. Y. Mizumoto, K.-I. Morita, \& M. Ohishi (San Francisco, CA: ASP), 139

Pérez-González, P. G., Kennicutt, Jr., R. C., Gordon, K. D., et al. 2006, ApJ, 648, 987

Pilbratt, G. L., Riedinger, J. R., Passvogel, T., et al. 2010, A\&A, 518, L1

Planck Collaboration, Ade, P. A. R., Aghanim, N., et al. 2011, A\&A, in press (arXiv:1101.2031)

Poglitsch, A., Waelkens, C., Geis, N., et al. 2010, A\&A, 518, L2

Quillen, A. C., \& Yukita, M. 2001, AJ, 121, 2095

Reddy, N. A., Erb, D. K., Pettini, M., Steidel, C. C., \& Shapley, A. E. 2010, ApJ, 712,1070

Regan, M. W., \& Vogel, S. N. 1995, ApJ, 452, L21

Relaño, M., Lisenfeld, U., Pérez-González, P. G., Vílchez, J. M., \& Battaner, E. 2007, ApJ, 667, L141

Rieke, G. H., Alonso-Herrero, A., Weiner, B. J., et al. 2009, ApJ, 692, 556

Rubin, R. H. 1968, ApJ, 154, 391

Salim, S., Rich, R. M., Charlot, S., et al. 2007, ApJS, 173, 267

Sauvage, M., \& Thuan, T. X. 1992, ApJ, 396, L69

Scaife, A. M. M., Nikolic, B., Green, D. A., et al. 2010, MNRAS, 406, L45

Schinnerer, E., Böker, T., Emsellem, E., \& Lisenfeld, U. 2006, ApJ, 649, 181

Schlegel, D. J., Finkbeiner, D. P., \& Davis, M. 1998, ApJ, 500, 525

Schmitt, H. R., Calzetti, D., Armus, L., et al. 2006, ApJ, 643, 173

Smith, J. D. T., Draine, B. T., Dale, D. A., et al. 2007, ApJ, 656, 770

Tabatabaei, F. S., Beck, R., Krause, M., et al. 2007, A\&A, 466, 509

Takeuchi, T. T., Buat, V., \& Burgarella, D. 2005, A\&A, 440, L17

Tammann, G. A. 1982, in NATO ASIC Proc. 90: Supernovae: A Survey of Current Research, ed. M. J. Rees \& R. J. Stoneham (Dordrecht: Reidel), 371

Tsai, C., Turner, J. L., Beck, S. C., et al. 2006, AJ, 132, 2383

Turner, J. L., \& Ho, P. T. P. 1983, ApJ, 268, L79

Turner, J. L., \& Ho, P. T. P. 1985, ApJ, 299, L77

Turner, J. L., \& Ho, P. T. P. 1994, ApJ, 421, 122

Turner, J. L., Ho, P. T. P., \& Beck, S. C. 1998, AJ, 116, 1212

van der Hulst, J. M., Kennicutt, R. C., Crane, P. C., \& Rots, A. H. 1988, A\&A, 195,38

Völk, H. J. 1989, A\&A, 218, 67

Walterbos, R. A. M., \& Greenawalt, B. 1996, ApJ, 460, 696

Weingartner, J. C., \& Draine, B. T. 2001, ApJ, 548, 296

Wu, H., Cao, C., Hao, C., et al. 2005, ApJ, 632, L79

Yun, M. S., Reddy, N. A., \& Condon, J. J. 2001, ApJ, 554, 803

Zhu, Y., Wu, H., Cao, C., \& Li, H. 2008, ApJ, 686, 155 